\title{
Matrix-Matched Iron-Oxide Laser Ablation ICP-MS U-Pb Geochronology Using Mixed Solution Standards
}

\author{
Liam Courtney-Davies ${ }^{1, *}$, Zhiyong Zhu ${ }^{1,2}$, Cristiana L. Ciobanu ${ }^{1}$, Benjamin P. Wade ${ }^{3}$, \\ Nigel J. Cook ${ }^{1}$, Kathy Ehrig ${ }^{4}$, Alexandre R. Cabral ${ }^{5}$ and Allen Kennedy ${ }^{6}$ \\ 1 School of Chemical Engineering, The University of Adelaide, Adelaide, SA 5005, Australia; \\ zhiyong.zhu@adelaide.edu.au (Z.Z); cristiana.ciobanu@adelaide.edu.au (C.L.C.); \\ nigel.cook@adelaide.edu.au (N.J.C.) \\ 2 State Key Laboratory for Mineral Deposits Research, Department of Earth Sciences, Nanjing University, \\ Nanjing 210093, China \\ 3 Adelaide Microscopy, The University of Adelaide, Adelaide, SA 5005, Australia; \\ Benjamin.wade@adelaide.edu.au \\ 4 BHP Billiton Olympic Dam, Adelaide, SA 5000, Australia; Kathy.J.Ehrig@bhpbilliton.com \\ 5 Lagerstätten und Rohstoffe, Technische Universität Clausthal, Adolph-Roemer-Str. 2A, \\ Clausthal-Zellerfeld D-38678, Germany; alexandre.cabral@tu-clausthal.de \\ 6 John de Laeter Centre, Curtin University of Technology, Bentley, WA 6102, Australia; \\ A.Kennedy@exchange.curtin.edu.au \\ * Correspondence: liam.courtney-davies@adelaide.edu.au; Tel.: +61-8-8313-4645
}

Academic Editor: Antonio Simonetti

Received: 18 May 2016; Accepted: 16 August 2016; Published: 23 August 2016

Abstract: U-Pb dating of the common iron-oxide hematite $\left(\alpha-\mathrm{Fe}_{2} \mathrm{O}_{3}\right)$, using laser-ablation inductively-coupled-plasma mass-spectrometry (LA-ICP-MS), provides unparalleled insight into the timing and processes of mineral deposit formation. Until now, the full potential of this method has been negatively impacted by the lack of suitable matrix-matched standards. To achieve matrix-matching, we report an approach in which a U-Pb solution and ablated material from 99.99\% synthetic hematite are simultaneously mixed in a nebulizer chamber and introduced to the ICP-MS. The standard solution contains fixed $\mathrm{U}$ - and $\mathrm{Pb}$-isotope ratios, calibrated independently, and aspiration of the isotopically homogeneous solution negates the need for a matrix-matched, isotopically homogenous natural iron-oxide standard. An additional advantage of using the solution is that the individual $\mathrm{U}-\mathrm{Pb}$ concentrations and isotope ratios can be adjusted to approximate that in the unknown, making the method efficient for dating hematite containing low ( 10 ppm) to high $(>1 \mathrm{wt} \%) \mathrm{U}$ concentrations. The above-mentioned advantage to this solution method results in reliable datasets, with arguably-better accuracy in measuring $\mathrm{U}-\mathrm{Pb}$ ratios than using GJ-1 Zircon as the primary standard, which cannot be employed for such low U concentrations. Statistical overlaps between ${ }^{207} \mathrm{~Pb} /{ }^{206} \mathrm{~Pb}$ weighted average ages (using GJ-1 Zircon) and U-Pb upper intercept ages (using the $\mathrm{U}-\mathrm{Pb}$ mixed solution method) of two samples from iron-oxide copper-gold (IOCG) deposits in South Australia demonstrate that, although fractionation associated with a non-matrix matched standard does occur when using GJ-1 Zircon as the primary standard, it does not impact the ${ }^{207} \mathrm{~Pb} /{ }^{206} \mathrm{~Pb}$ or upper intercept age. Thus, GJ-1 Zircon can be considered reliable for dating hematite using LA-ICP-MS. Downhole fractionation of ${ }^{206} \mathrm{~Pb} /{ }^{238} \mathrm{U}$ is observed to occur in spot analyses of hematite. The use of rasters in future studies will hopefully minimize this problem, allowing for matrix-matched data. Using the mixed-solution method in this study, we have validated a published hematite $\mathrm{Pb}-\mathrm{Pb}$ age for Olympic Dam, and provide a new age (1604 $\pm 11 \mathrm{Ma})$ for a second deposit in the same province. These ages are further evidence that the IOCG mineralizing event is tied to large igneous province (LIP) magmatism in the region at $\sim 1.6 \mathrm{Ga}$.

Keywords: iron-oxide; U-Pb geochronology; matrix-matched standard; LA-ICP-MS 


\section{Introduction}

Laser-ablation inductively-coupled plasma mass-spectrometry (LA-ICP-MS allows the user to rapidly obtain accurate geochronological data [1,2]. The caveat to this is the requirement of certified matrix-matched reference materials, which do not exist for a large number of potentially dateable minerals (unless it can be demonstrated that the effects of using a non-matrix-matched standard are smaller than the propagated errors). This includes the common iron-oxide, hematite $\left(\alpha-\mathrm{Fe}_{2} \mathrm{O}_{3}\right)$, which, until now, has only been dated by the U-Pb method using the reference material GJ-1 Zircon [1] as the primary external standard [3]. Analysis of uranium-bearing (up to several wt $\% \mathrm{U}$ ) hematite from Olympic Dam (OD), South Australia, returned dates for hematite mineralization in two samples $(1577 \pm 5$ and $1590 \pm 8 \mathrm{Ma}$ ) [3]. These are in good agreement with $\mathrm{U} / \mathrm{Pb}$ zircon ages from OD [4,5]. Ciobanu et al. (2013) [3], thus, argued that the utilization of the GJ-1 Zircon standard is sufficiently reliable for exploratory Fe-oxide geochronological applications.

The analytical approach used by Ciobanu et al. (2013) [3] did not employ a matrix-matched standard, and, thus, only ${ }^{207} \mathrm{~Pb} /{ }^{206} \mathrm{~Pb}$ dates could be considered reliable due to the relatively low matrix-dependent fractionation effects on $\mathrm{Pb}-\mathrm{Pb}$ ratios. Accurate $\mathrm{U} / \mathrm{Pb}$ ages could not be well constrained, as the matrix effects on the measured $\mathrm{U} / \mathrm{Pb}$ ratios in hematite were largely unknown when standardizing to a non-matrix-matched standard, such as zircon. These matrix effects include phenomena such as laser-induced elemental fractionation, resultant plasma loading and ionization [5-7]. The present study aims at circumventing this problem by using an alternative, mixed solution-solid type of standard for $\mathrm{U} / \mathrm{Pb}$ dating using LA-ICP-MS. This concept has been tested successfully for $\mathrm{U} / \mathrm{Pb}$ dating of zircon and baddeleyite [8-10], and non-matrix-matched trace element determination [11,12], and is here applied to dating of U-bearing hematite for the first time. The aims of this study were to: Evaluate the potential of hematite as a $\mathrm{U}-\mathrm{Pb}$ geochronometer; to assess the effect of "matrix effects" when using a non-matrix matched standard, such as zircon, by comparison with the data of Ciobanu et al. (2013) [3]; to test whether the method gives reliable ages for hematite with low U concentrations; and to provide an "easy-and-ready to use" method for $\mathrm{U} / \mathrm{Pb}$ dating of hematite.

\section{Experimental Methodology}

\subsection{Instrumentation}

Isotope measurements were determined using a Resonetics 193 ArF M-50 Excimer laser ablation system coupled to an Agilent 7700s ICP-MS, housed at Adelaide Microscopy, University of Adelaide. Analysis parameters included a constant repetition rate of $5 \mathrm{~Hz}$, laser fluence of $4.3-9.4 \mathrm{~J} / \mathrm{cm}^{2}$, and a beam diameter set between 18 and $60 \mu \mathrm{m}$ (spot analyses) depending on the size of the analyzed U-bearing zones within the hematite grains and their U concentrations. Spot analyses were chosen due to the small size of the hematite grains, which do not have large enough areas of high $\mathrm{U}$ zonation to use line rastering. Data were collected using time-resolved acquisition in fast peak-jumping mode and calculations were carried out using the data reduction software GLITTER [13]. Isotope ratios are presented uncorrected for common lead, with concordia plots generated using Isoplot/Ex 3.75 [14]. Instrument optimization and calibration was performed using NIST-610 standard glass (National Institute of Standards and Technology, U.S. Department of Commerce). Table 1 provides instrument and analysis parameters. 
Table 1. Instrument and analysis parameters.

\begin{tabular}{|c|c|c|c|}
\hline Laser Ablation System & & ICP-MS & \\
\hline Type & ArF Excimer & Type & Agilent 7700 \\
\hline Wavelength & $193 \mathrm{~nm}$ & Makeup gas & Argon \\
\hline Repetition Rate & $5 \mathrm{~Hz}$ & Peltier chamber temperature & $2 \mathrm{C}$ \\
\hline Spot diameter & $18-60 \mu \mathrm{m}$ & Plasma RF power & $1550 \mathrm{~W}$ \\
\hline Solution standard & $\mathrm{U}-\mathrm{Pb}$ & Acquisition mode & TRA \\
\hline He gas flow & $0.55 \mathrm{~L} \cdot \mathrm{min}^{-1}$ & Detector mode & Dual Range \\
\hline Laser Fluence & $4.3-9.4 \mathrm{~J} / \mathrm{cm}^{2}$ & Dwell Time & \\
\hline Solution Nebulization & & ${ }^{204} \mathrm{~Pb}$ & $10 \mathrm{~ms}$ \\
\hline Nebulizer & Quartz $250 \mathrm{uL} / \mathrm{min}$ & ${ }^{206} \mathrm{~Pb}$ & $50 \mathrm{~ms}$ \\
\hline Ar carrier gas flow & $0.5 \mathrm{~L} \cdot \mathrm{min}^{-1}$ & ${ }^{207} \mathrm{~Pb}$ & $40 \mathrm{~ms}$ \\
\hline \multirow[t]{2}{*}{ Spray chamber } & Custom Cyclonic & ${ }^{208} \mathrm{~Pb}$ & $30 \mathrm{~ms}$ \\
\hline & & ${ }^{238} \mathrm{U}$ & $30 \mathrm{~ms}$ \\
\hline
\end{tabular}

\subsection{Experimental Procedure}

A typical measurement of the "standard" in this experiment comprises aspiration of a liquid, represented by a $\mathrm{U}-\mathrm{Pb}$ solution with known isotopic ratios in $2 \% \mathrm{HNO}_{3}$, combined with the ablated aerosol of a pure hematite sample, mounted in a 1-inch diameter epoxy block. Mixing was carried out in a spray chamber, in which the ablated hematite aerosol was fed and mixed with the aspirated and nebulized $\mathrm{U}-\mathrm{Pb}$ solution. Analysis of unknowns comprised mixing of the ablated unknown aerosol with a pure $2 \% \mathrm{HNO}_{3}$ solution to ensure the same liquid plasma loading between the standard and unknown. Between analysis of standards and unknowns, the solution line was washed with aspirated $5 \% \mathrm{HNO}_{3}$ solution. Figure 1 shows the LA-ICP-MS system setup.

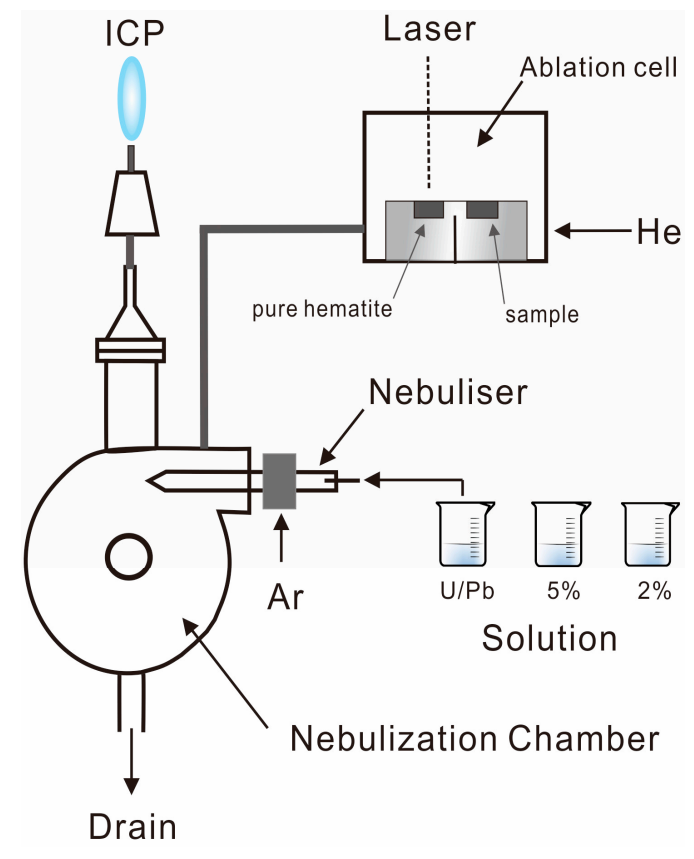

Figure 1. Schematic of the sample introduction system displaying the laser-ablation inductivelycoupled plasma-mass spectrometry (LA-ICP-MS) system connected to the U-Pb solution and blank $\mathrm{HNO}_{3}$ solutions. 


\subsection{Reagents and Standard Material}

The pure hematite analyzed as part of the standard analysis comprised a laboratory-grade synthetic hematite (3-12 mm in size, $\geq 99.98 \%$ trace metals basis; Sigma Aldrich Pty. Ltd., St. Louis, $\mathrm{MO}, \mathrm{USA})$. The $\mathrm{U}-\mathrm{Pb}$ calibration solution was made via mixing individual $\mathrm{U}$ and $\mathrm{Pb}$ single element standard solutions in $2 \% \mathrm{HNO}_{3}$ diluted with Milli-Q water. The $\mathrm{Pb}$ isotope ratios of the resultant solution were measured by MC-ICP-MS at Nanjing University, China (Table S1), using added thallium as an internal isotopic standard [15] to correct for mass-dependent isotopic fractionation. Absolute $\mathrm{U} / \mathrm{Pb}$ concentrations were calibrated via solution ICP-MS on an Agilent $7500 \mathrm{cs}$ instrument at Adelaide Microscopy (Table S2). Depending on the U concentration of the sample being analyzed, a stronger or weaker $\mathrm{U}-\mathrm{Pb}$ solution was made by dilution with $2 \% \mathrm{HNO}_{3}$. The criteria for using different solution strengths were based on the counts-per-second (CPS) registered by the LA-ICP-MS when optimizing the machine parameters.

\subsection{Stability and Precision of the Mass Spectrometer}

$\mathrm{U}-\mathrm{Pb}$ mixed standard solutions avoid the potential heterogeneity problems encountered by solid standards. The measured ${ }^{206} \mathrm{~Pb} /{ }^{238} \mathrm{U}$ ratio of our solution standard is shown in Figure $2 \mathrm{~A}$. To ensure the ${ }^{238} \mathrm{U}$ sensitivity of the unknowns is matched to the standard, pre-ablation of the sample was carried out to assess the ${ }^{238} \mathrm{U}$ CPS. The ${ }^{238} \mathrm{U}$ concentration of the standard was then adjusted appropriately. In our experiment, two concentrations of $\mathrm{U}-\mathrm{Pb}$ solution ( 1 and $12 \mathrm{ppb}$ ) were employed. The 12-ppb $\mathrm{U}-\mathrm{Pb}$ mixed solution was used for hematite containing hundreds of ppm $\mathrm{U}$, and the 1-ppb solution was used to calibrate samples that were lower in $U$ (tens of ppm). Each analytical run of 15 unknowns was bracketed by two solution standards.
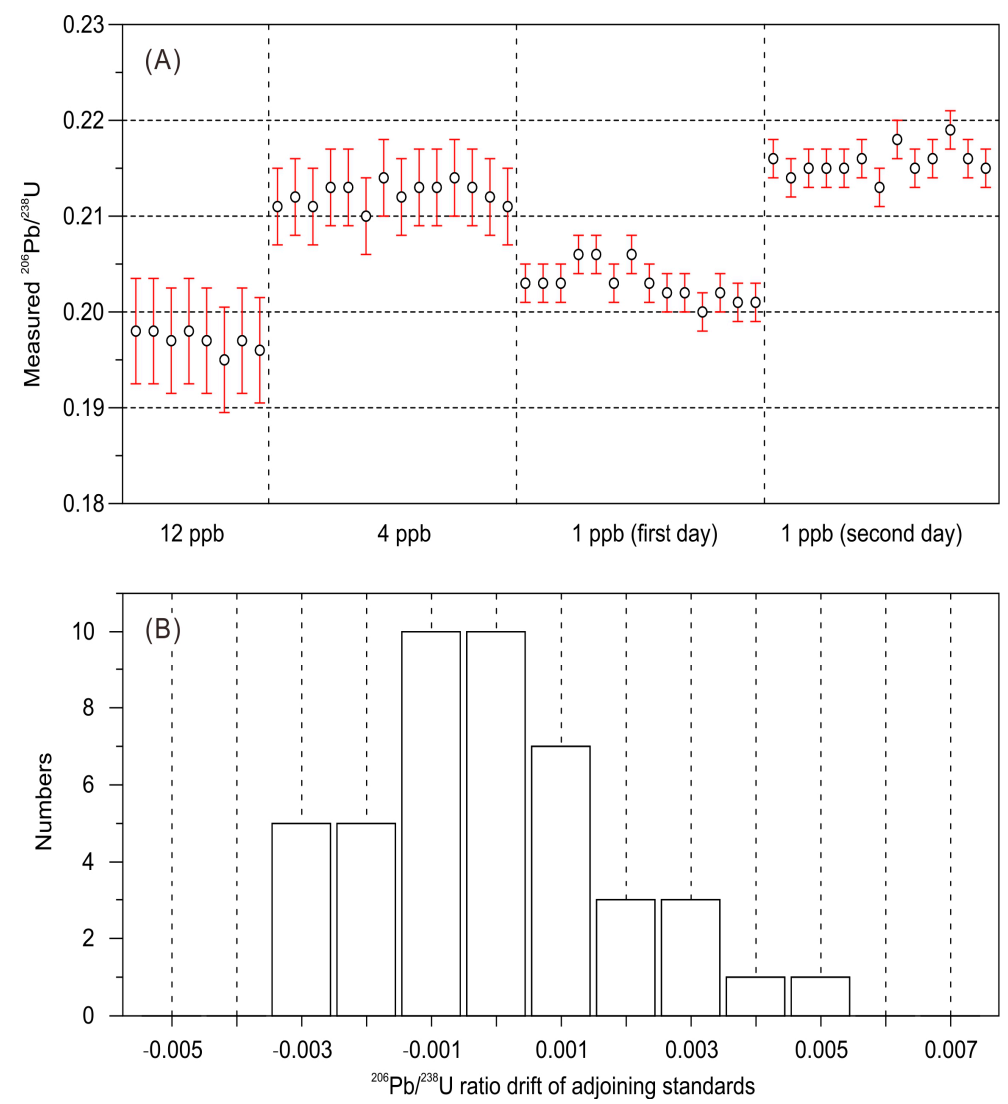

Figure 2. (A) Measured ${ }^{206} \mathrm{~Pb} /{ }^{238} \mathrm{U}$ ratios of solution standards with different concentrations; (B) ${ }^{206} \mathrm{~Pb} /{ }^{238} \mathrm{U}$ drifts of adjoining standards. The error bars represent 1 sigma standard error. 
The total integration time was $60 \mathrm{~s}$, which incorporated $25 \mathrm{~s}$ for background collection followed by $35 \mathrm{~s}$ for laser firing. This was followed by $40 \mathrm{~s}$ for washing. The measured ${ }^{206} \mathrm{~Pb} /{ }^{238} \mathrm{U}$ drift over the 23 -min period of each run sequence is less than \pm 0.003 , which accounts for only $1.5 \%$ of the measured ratio (Figure $2 \mathrm{~B}$ ). Although the measured ${ }^{206} \mathrm{~Pb} /{ }^{238} \mathrm{U}$ ratio for the same solution can vary day-to-day, the variation within one run is minimal (Figure 2B), allowing the acquisition of high-precision data. In particular, the stability of the 1-ppb mixing solution was still very efficient, even for especially low U samples. Fifteen spots were analyzed on NIST610, immediately following analysis of OD10-4 using just the solution standard calibration method, which gave a $1 \sigma$ error for the ${ }^{206} \mathrm{~Pb} /{ }^{238} \mathrm{U}$ age of $3-4 \mathrm{Ma}$ $(<1 \%)$, testifying to the stability of the measured signals and instrument at the time of analysis.

\subsection{Calibration Process}

The ${ }^{207} \mathrm{~Pb} /{ }^{206} \mathrm{~Pb}$ ratio $(0.866312)$ of the solution standard was measured using MC-ICP-MS with uncertainties of $\sim 0.12 \%$. The ${ }^{206} \mathrm{~Pb} /{ }^{238} \mathrm{U}$ ratios $(0.286356)$ were calculated from Q-ICP-MS U/Pb concentration data and MC-ICP-MS Pb isotope ratios; this is expanded upon in Supplementary Text File S1. We assume that the uncertainties of ${ }^{206} \mathrm{~Pb}$ and ${ }^{238} \mathrm{U}$ concentrations are $1 \%$; and the propagated 1 sigma deviation of ${ }^{206} \mathrm{~Pb} /{ }^{238} \mathrm{U}$ was $1.4 \%$. The uncertainties are then defined in GLITTER (GEMOC Laser ICPMS Total Trace Element Reduction software, Macquarie University, Sydney, Australia) to calibrate the samples. The two ratios from the solution standard were used to calibrate the ${ }^{207} \mathrm{~Pb} /{ }^{206} \mathrm{~Pb}$ and the ${ }^{206} \mathrm{~Pb} /{ }^{238} \mathrm{U}$ ratios of the samples respectively, while the final ${ }^{207} \mathrm{~Pb} /{ }^{235} \mathrm{U}$ ratios of the samples were calculated from the calibrated ${ }^{207} \mathrm{~Pb} /{ }^{206} \mathrm{~Pb}$ and ${ }^{206} \mathrm{~Pb} /{ }^{238} \mathrm{U}$.

\section{Sample Selection}

Hematite samples used in this study are from iron-oxide copper gold (IOCG) deposits in South Australia (Figure 3), and an analogous IOCG province, the Carajás mineral province, Brazil. The Australian samples (i.e., OD10-4 from Olympic Dam and PH-93 from a potentially different tectonic setting within the Olympic $\mathrm{Cu}$-Au Province) contain thousands of ppm to a few wt \% U, while the Brazilian sample (CUR-002) has orders of magnitude lower $\mathrm{U}$, but is higher in $\mathrm{W}$ (hundreds ppm). Prior to LA-ICP-MS analysis, polished blocks were imaged on an FEI Quanta 450 FEG ESEM, using a back-scattered electron detector optimized to reveal the presence of elevated $U$ and grain-scale zonation in hematite. The samples are all compositionally zoned in uranium (Figure 4), and it is such zones that were targeted for dating.

Sample OD10-4 represents a high-grade ore where the high-U hematite is embedded in bornite (Figure 4A). This is one of ten polished blocks prepared from the same hand specimen, petrographically characterized and analyzed by Ciobanu et al. (2013) [3], who obtained a ${ }^{207} \mathrm{~Pb} /{ }^{206} \mathrm{~Pb}$ weighted average age of $1577 \pm 5 \mathrm{Ma}$. Sample PH-93 represents hematite breccia cemented with chalcopyrite, and is sourced from an IOCG deposit to the north-nest of Olympic Dam. Although similar in terms of hematite textures to the Olympic Dam material, the high-U zones in PH-93 are coarser (Figure 4B). This sample was also analyzed in this study using GJ-1 Zircon as the primary standard to allow comparison between the two methods. Sample CUR-002 was dated to test the reliability of the mixed solution method with a provincially non-related sample. The sample is from a vein filled with aggregates of coarse lamellar hematite, displaying similar but weaker zoning compared to the two Australian specimens (Figure 4C,D). The vein from which sample CUR-002 was taken crosscuts quartzite and is located some $30 \mathrm{~km}$ east north-east of the $1.88 \mathrm{Ga}$ Cigano granite [16], in the ridge that marks the Cinzento strike-slip system of the Carajás mineral province [17].

In addition to providing direct comparison between analysis using GJ-1 Zircon and mixed solution standards, the samples were chosen to assess the age obtained for high-grade ore at OD, to provide a hematite age for associated mineralization elsewhere in the Olympic $\mathrm{Cu}-\mathrm{Au}$ Province, and to test the application of the method on low-U samples. Ultimately, the choice of samples was designed to illustrate how the method introduced here might find broad application throughout the IOCG province in South Australia and elsewhere. 


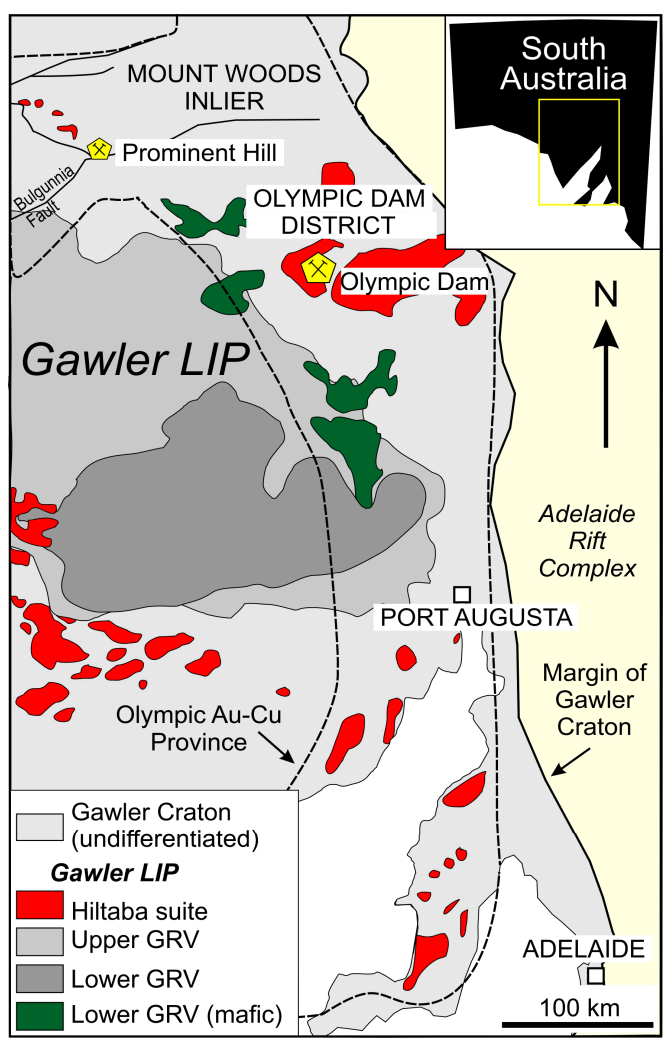

Figure 3. Sketch map showing the location of the Olympic Cu-Au Province within South Australia, as well as exposed Gawler Range Volcanics and Hiltaba Suite intrusive rocks.

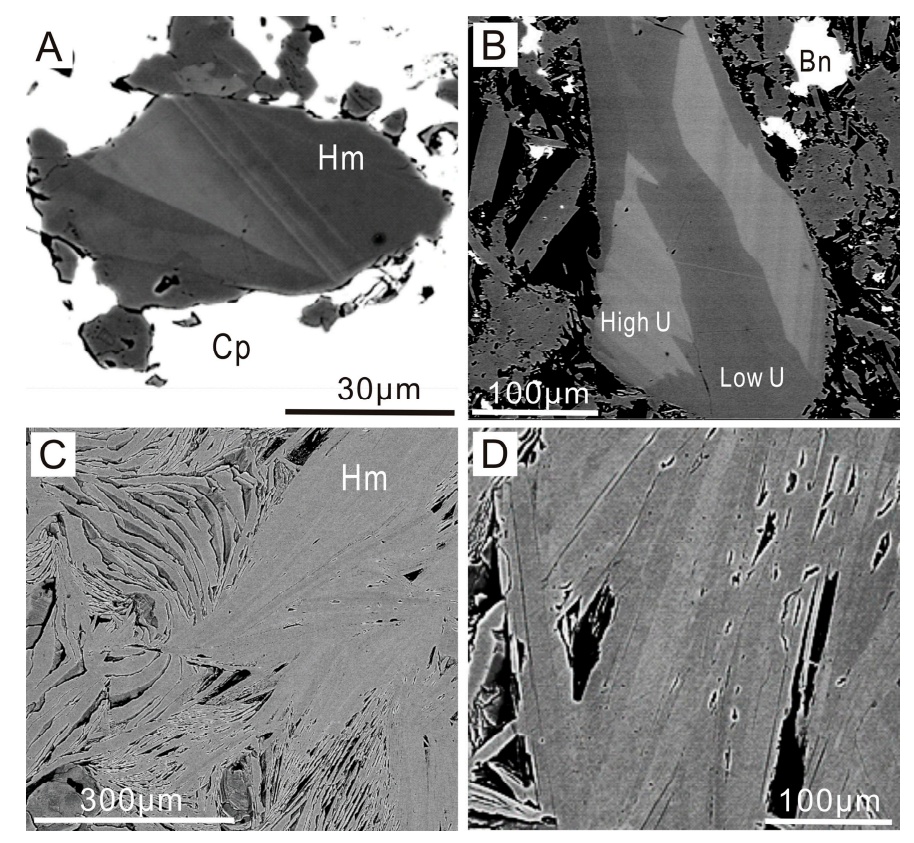

Figure 4. Back scattered electron images of U-bearing zoned hematite. (A,B) Hematite (Hm) displaying oscillatory and sectorial zoning expressed as high-U from Olympic Dam (A) and a second sample from another IOCG deposit in the Olympic Cu-Au Province (B) note differences in size, and coarser zones for the latter. Additionally note association with sulphides, bornite (Bn) and chalcopyrite (Cp), respectively; (C,D) low-U hematite from Carajás (CUR-002) showing aggregates of hematite with zones containing $\mathrm{U}$ and $\mathrm{W}$ in the centers of the lamellae (brighter on BSE images). 


\section{4. $\mathrm{U} / \mathrm{Pb}$ Geochronology Results}

Sample OD10-4 (Table 2, Figure 5) produced a U/Pb upper intercept date of $1595 \pm 18 \mathrm{Ma}$ and was anchored to a lower intercept of $0 \pm 40 \mathrm{Ma}$ using an $18 \mu \mathrm{m}$ spot size and a 12-ppb mixed solution. None of the spots analyzed in this sample have been excluded from the processed dataset. The data ranges from $100 \%$ to $112 \%$ concordance, with the discordancy either due to recent $\mathrm{Pb}$ loss, or, more likely, due to laser induced elemental fractionation; points plotting above the concordia relate to U loss. Although the error is relatively high, the low amount of points analyzed and inclusion of all points attests to the reliability of the method to generate coherent ages.

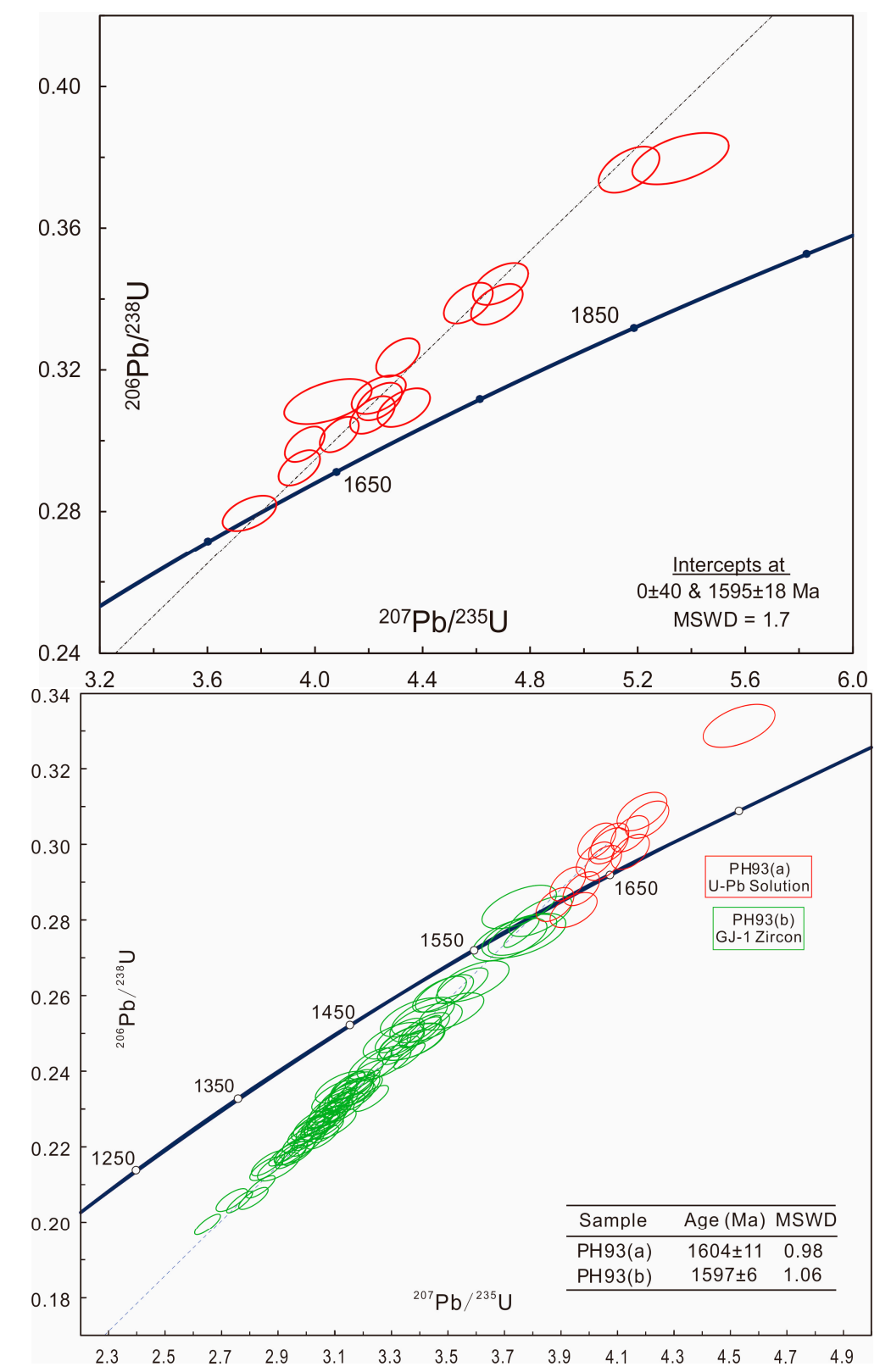

Figure 5. Conventional concordia plots displaying analyzed grains of high-U hematite from the Olympic Province. The first concordia displays sample OD10-4 analyzed through the U-Pb solution method. The second concordia overlays data from sample PH93 collected through the U-Pb solution method (a) and GJ-1 Zircon (b). Data are given in Tables 2-4. Note, all U-Pb solution data plots on or above concordia, whereas all GJ-1 Zircon data plots on or below concordia. 
Table 2. Geochronological data for zoned hematite (sample OD10-4) using mixed-solution method.

\begin{tabular}{|c|c|c|c|c|c|c|c|c|c|c|c|c|c|c|c|c|c|c|c|c|}
\hline $\begin{array}{l}\text { Spot } \\
\text { No. }\end{array}$ & ${ }^{207} \mathrm{~Pb} /{ }^{206} \mathrm{~Pb}$ & $1 \sigma$ & ${ }^{206} \mathrm{~Pb} /{ }^{238} \mathrm{U}$ & $1 \sigma$ & ${ }^{207} \mathrm{~Pb} /{ }^{235} \mathrm{U}$ & $1 \sigma$ & ${ }^{207} \mathrm{~Pb} /{ }^{206} \mathrm{~Pb}$ & $1 \sigma$ & ${ }^{206} \mathrm{~Pb} /{ }^{238} \mathrm{U}$ & $1 \sigma$ & ${ }^{207} \mathrm{~Pb} /{ }^{235} \mathrm{U}$ & $1 \sigma$ & $\begin{array}{c}\text { Concordancy } \\
(\%)\end{array}$ & ${ }^{204} \mathrm{~Pb}$ & ${ }^{206} \mathrm{~Pb}$ & ${ }^{207} \mathrm{~Pb}$ & ${ }^{208} \mathrm{~Pb}$ & ${ }^{238} \mathrm{U}$ & ${ }^{204} \mathrm{~Pb} /{ }^{207} \mathrm{~Pb}$ & Inc/Exc \\
\hline 1 & 0.0975 & 0.0019 & 0.2795 & 0.0033 & 3.7565 & 0.0662 & 1577 & 35 & 1589 & 17 & 1584 & 16 & 100 & 5 & 33,155 & 3283 & 137 & 171,602 & 0.0015 & Inc \\
\hline 2 & 0.0978 & 0.0013 & 0.2923 & 0.0033 & 3.9418 & 0.0510 & 1582 & 24 & 1653 & 17 & 1622 & 12 & 102 & 3 & 76,344 & 7580 & 91 & 377,715 & 0.0004 & Inc \\
\hline 3 & 0.0966 & 0.0012 & 0.3235 & 0.0036 & 4.3083 & 0.0533 & 1560 & 23 & 1807 & 18 & 1695 & 11 & 107 & 0 & 91,744 & 8999 & 52 & 410,380 & 0.0000 & Inc \\
\hline 4 & 0.0944 & 0.0029 & 0.3111 & 0.0042 & 4.0485 & 0.1076 & 1516 & 56 & 1746 & 21 & 1644 & 24 & 106 & 14 & 12,125 & 1161 & 176 & 56,402 & 0.0121 & Inc \\
\hline 5 & 0.0961 & 0.0012 & 0.2990 & 0.0033 & 3.9618 & 0.0489 & 1550 & 23 & 1686 & 17 & 1626 & 11 & 104 & 17 & 98,322 & 9589 & 119 & 475,862 & 0.0018 & Inc \\
\hline 6 & 0.1002 & 0.0014 & 0.3385 & 0.0038 & 4.6762 & 0.0635 & 1628 & 25 & 1879 & 19 & 1763 & 13 & 107 & 0 & 57,251 & 5819 & 169 & 244,735 & 0.0000 & Inc \\
\hline 7 & 0.1024 & 0.0025 & 0.3796 & 0.0048 & 5.3595 & 0.1186 & 1668 & 45 & 2075 & 23 & 1878 & 21 & 110 & 0 & 10,321 & 1072 & 185 & 39,352 & 0.0000 & Inc \\
\hline 8 & 0.0988 & 0.0013 & 0.3111 & 0.0035 & 4.2398 & 0.0554 & 1602 & 24 & 1746 & 18 & 1682 & 12 & 104 & 0 & 68,567 & 6873 & 202 & 319,022 & 0.0000 & Inc \\
\hline 9 & 0.0995 & 0.0013 & 0.3073 & 0.0035 & 4.2137 & 0.0550 & 1614 & 24 & 1727 & 18 & 1677 & 12 & 103 & 5 & 62,854 & 6338 & 539 & 296,105 & 0.0008 & Inc \\
\hline 10 & 0.0982 & 0.0016 & 0.3130 & 0.0036 & 4.2370 & 0.0666 & 1590 & 31 & 1755 & 18 & 1681 & 14 & 104 & 3 & 31,642 & 3148 & 243 & 146,337 & 0.0010 & Inc \\
\hline 11 & 0.0983 & 0.0011 & 0.3017 & 0.0033 & 4.0906 & 0.0478 & 1593 & 21 & 1700 & 17 & 1652 & 11 & 103 & 7 & 205,525 & 20,481 & 174 & 986,231 & 0.0003 & Inc \\
\hline 12 & 0.0988 & 0.0015 & 0.3442 & 0.0039 & 4.6900 & 0.0679 & 1602 & 28 & 1907 & 20 & 1765 & 14 & 108 & 11 & 44,410 & 4447 & 305 & 186,834 & 0.0025 & Inc \\
\hline 13 & 0.0996 & 0.0015 & 0.3765 & 0.0043 & 5.1679 & 0.0747 & 1616 & 28 & 2060 & 21 & 1847 & 14 & 112 & 0 & 42,844 & 4321 & 79 & 164,804 & 0.0000 & Inc \\
\hline 14 & 0.1015 & 0.0016 & 0.3093 & 0.0036 & 4.3303 & 0.0647 & 1652 & 29 & 1737 & 18 & 1699 & 14 & 102 & 0 & 30,706 & 3158 & 229 & 143,785 & 0.0000 & Inc \\
\hline 15 & 0.0978 & 0.0013 & 0.3389 & 0.0038 & 4.5702 & 0.0602 & 1583 & 25 & 1881 & 19 & 1744 & 12 & 108 & 23 & 51,642 & 5115 & 1491 & 220,769 & 0.0045 & Inc \\
\hline
\end{tabular}

Table 3. Geochronological data for zoned hematite (sample PH-93) using mixed-solution method.

\begin{tabular}{|c|c|c|c|c|c|c|c|c|c|c|c|c|c|c|c|c|c|c|c|c|}
\hline Spot No. & ${ }^{207} \mathrm{~Pb} /{ }^{206} \mathrm{~Pb}$ & $1 \sigma$ & ${ }^{206} \mathrm{~Pb} /{ }^{238} \mathrm{U}$ & $1 \sigma$ & ${ }^{207} \mathrm{~Pb} /{ }^{235} \mathrm{U}$ & $1 \sigma$ & ${ }^{207} \mathrm{~Pb} /{ }^{206} \mathrm{~Pb}$ & $1 \sigma$ & ${ }^{206} \mathrm{~Pb} /{ }^{238} \mathrm{U}$ & $1 \sigma$ & ${ }^{207} \mathrm{~Pb} /{ }^{235} \mathrm{U}$ & $1 \sigma$ & Concordancy (\%) & ${ }^{204} \mathrm{~Pb}$ & ${ }^{206} \mathrm{~Pb}$ & ${ }^{207} \mathrm{~Pb}$ & ${ }^{208} \mathrm{~Pb}$ & ${ }^{238} \mathrm{U}$ & ${ }^{204} \mathrm{~Pb} /{ }^{207} \mathrm{~Pb}$ & Inc/Exc \\
\hline 1 & 0.0969 & 0.0011 & 0.3013 & 0.0032 & 4.0267 & 0.0444 & 1566 & 20 & 1698 & 16 & 1640 & 10 & 104 & 0 & 115,237 & 11,217 & 2 & 524,174 & 0.00000 & Inc \\
\hline 2 & 0.1008 & 0.0011 & 0.2984 & 0.0031 & 4.1449 & 0.0446 & 1638 & 19 & 1683 & 16 & 1663 & 10 & 101 & 0 & 183,960 & 18,611 & - & 845,094 & 0.00000 & Inc \\
\hline 3 & 0.0991 & 0.0010 & 0.3032 & 0.0032 & 4.1430 & 0.0443 & 1607 & 19 & 1707 & 16 & 1663 & 10 & 103 & 6 & 213,122 & 21,209 & 8 & 963,706 & 0.00028 & Inc \\
\hline 4 & 0.0994 & 0.0010 & 0.2954 & 0.0031 & 4.0487 & 0.0431 & 1613 & 19 & 1669 & 16 & 1644 & 10 & 101 & 0 & 265,097 & 26,461 & 5 & $1,230,702$ & 0.00000 & Inc \\
\hline 5 & 0.0980 & 0.0011 & 0.3013 & 0.0032 & 4.0726 & 0.0443 & 1587 & 20 & 1698 & 16 & 1649 & 10 & 103 & 0 & 130,179 & 12,815 & 0 & 592,727 & 0.00000 & Inc \\
\hline 6 & 0.0984 & 0.0011 & 0.2964 & 0.0031 & 4.0208 & 0.0443 & 1594 & 20 & 1673 & 16 & 1638 & 10 & 102 & 6 & 141,507 & 13,980 & 4 & 655,082 & 0.00043 & Inc \\
\hline 7 & 0.0998 & 0.0011 & 0.2885 & 0.0030 & 3.9717 & 0.0427 & 1621 & 19 & 1634 & 16 & 1628 & 10 & 100 & 0 & 167,315 & 16,772 & 0 & 795,856 & 0.00000 & Inc \\
\hline 8 & 0.0982 & 0.0015 & 0.3092 & 0.0034 & 4.1860 & 0.0581 & 1590 & 27 & 1737 & 17 & 1671 & 13 & 104 & 9 & 19,282 & 1900 & 4 & 85,593 & 0.00474 & Inc \\
\hline 9 & 0.0982 & 0.0010 & 0.2898 & 0.0030 & 3.9241 & 0.0417 & 1590 & 19 & 1641 & 16 & 1619 & 10 & 101 & 0 & 326,254 & 32,167 & 18 & $1,545,714$ & 0.00000 & Inc \\
\hline 10 & 0.0990 & 0.0011 & 0.2843 & 0.0030 & 3.8787 & 0.0443 & 1605 & 21 & 1613 & 15 & 1609 & 10 & 100 & 13 & 134,769 & 13,391 & 2 & 651,199 & 0.00097 & Inc \\
\hline 11 & 0.0983 & 0.0011 & 0.3000 & 0.0032 & 4.0680 & 0.0463 & 1593 & 21 & 1691 & 16 & 1648 & 10 & 103 & 6 & 133,301 & 13,161 & 0 & 610,394 & 0.00046 & Inc \\
\hline 12 & 0.0993 & 0.0012 & 0.3070 & 0.0033 & 4.2042 & 0.0508 & 1611 & 23 & 1726 & 17 & 1675 & 11 & 103 & 11 & 107,235 & 10,692 & 21 & 479,948 & 0.00103 & Inc \\
\hline 13 & 0.0989 & 0.0020 & 0.3322 & 0.0038 & 4.5293 & 0.0839 & 1604 & 38 & 1849 & 19 & 1736 & 17 & 106 & 0 & 20,203 & 2006 & 6 & 83,611 & 0.00000 & Inc \\
\hline 14 & 0.1012 & 0.0015 & 0.2828 & 0.0031 & 3.9446 & 0.0549 & 1646 & 27 & 1605 & 16 & 1623 & 13 & 99 & 6 & 35,223 & 3578 & 19 & 171,296 & 0.00168 & Inc \\
\hline
\end{tabular}


Table 4. Geochronological data for zoned hematite (sample PH-93) using GJ-1 Zircon as standard, U units are in net integrated counts/s.

\begin{tabular}{|c|c|c|c|c|c|c|c|c|c|c|c|c|c|c|c|c|c|c|c|c|c|}
\hline $\begin{array}{l}\text { Spot } \\
\text { No. }\end{array}$ & ${ }^{207} \mathrm{~Pb} /{ }^{206} \mathrm{~Pb}$ & $1 \sigma$ & ${ }^{206} \mathrm{~Pb} /{ }^{238} \mathrm{U}$ & $1 \sigma$ & ${ }^{207} \mathrm{~Pb} /{ }^{235} \mathrm{U}$ & $1 \sigma$ & ${ }^{207} \mathrm{~Pb} /{ }^{206} \mathrm{~Pb}$ & $1 \sigma$ & ${ }^{206} \mathrm{~Pb} /{ }^{238} \mathrm{U}$ & $1 \sigma$ & ${ }^{207} \mathrm{~Pb} /{ }^{235} \mathrm{U}$ & $1 \sigma$ & $\begin{array}{c}\text { Concordancy } \\
(\%)\end{array}$ & ${ }^{204} \mathrm{~Pb}$ & ${ }^{206} \mathrm{~Pb}$ & ${ }^{207} \mathrm{~Pb}$ & ${ }^{208} \mathrm{~Pb}$ & ${ }^{238} \mathrm{U}$ & ${ }^{204} \mathrm{~Pb} /{ }^{207} \mathrm{~Pb}$ & Inc/Exc & Notes \\
\hline 1 & 0.0974 & 0.0016 & 0.2491 & 0.0030 & 3.3457 & 0.0563 & 1575.6 & 31 & 1434 & 15 & 1492 & 13 & 91 & 0 & 358,124 & 35,083 & 0 & $1,571,490$ & 0.0000 & Inc & \\
\hline 2 & 0.0983 & 0.0015 & 0.2288 & 0.0025 & 3.1016 & 0.0457 & 1592.9 & 28 & 1328 & 13 & 1433 & 11 & 83 & 34 & $1,679,299$ & 165,047 & 57 & $7,631,865$ & 0.0002 & Inc & \\
\hline 3 & 0.0993 & 0.0017 & 0.2507 & 0.0030 & 3.4310 & 0.0585 & 1611.0 & 31 & 1442 & 15 & 1512 & 13 & 90 & 47 & $4,388,089$ & 436,897 & 1904 & $19,018,996$ & 0.0001 & Inc & \\
\hline 4 & 0.0991 & 0.0012 & 0.2761 & 0.0025 & 3.7704 & 0.0405 & 1606.6 & 22 & 1572 & 13 & 1587 & 9 & 98 & 26 & 641,549 & 63,493 & 119 & $2,066,412$ & 0.0004 & Inc & \\
\hline 5 & 0.0994 & 0.0015 & 0.2301 & 0.0026 & 3.1542 & 0.0462 & 1613.5 & 27 & 1335 & 13 & 1446 & 11 & 83 & 15 & $3,612,039$ & 359,811 & 311 & $16,437,574$ & 0.0000 & Inc & \\
\hline 6 & 0.0977 & 0.0016 & 0.2363 & 0.0026 & 3.1819 & 0.0508 & 1580.2 & 31 & 1367 & 14 & 1453 & 12 & 87 & 26 & 626,242 & 61,641 & 34 & $2,629,886$ & 0.0004 & Inc & \\
\hline 7 & 0.0988 & 0.0016 & 0.2268 & 0.0026 & 3.0890 & 0.0491 & 1601.8 & 29 & 1318 & 14 & 1430 & 12 & 82 & 12 & $1,057,774$ & 105,597 & 246 & $5,011,429$ & 0.0001 & Inc & \\
\hline 8 & 0.0994 & 0.0015 & 0.2347 & 0.0026 & 3.2155 & 0.0464 & 1612.7 & 27 & 1359 & 13 & 1461 & 11 & 84 & 13 & 686,076 & 68,084 & 317 & $2,983,425$ & 0.0002 & Inc & \\
\hline 9 & 0.0983 & 0.0012 & 0.2317 & 0.0021 & 3.1384 & 0.0343 & 1591.3 & 23 & 1343 & 11 & 1442 & 8 & 84 & 36 & 481,616 & 47,324 & 27 & $1,806,129$ & 0.0008 & Inc & \\
\hline 10 & 0.0991 & 0.0013 & 0.2452 & 0.0025 & 3.3499 & 0.0422 & 1607.3 & 25 & 1414 & 13 & 1493 & 10 & 88 & 21 & 897,793 & 88,769 & 126 & $3,483,487$ & 0.0002 & Inc & \\
\hline 11 & 0.0992 & 0.0014 & 0.2500 & 0.0027 & 3.4188 & 0.0485 & 1609.3 & 27 & 1438 & 14 & 1509 & 11 & 89 & 43 & $1,236,666$ & 122,600 & 265 & $5,024,110$ & 0.0004 & Inc & \\
\hline 12 & 0.0985 & 0.0014 & 0.2311 & 0.0024 & 3.1389 & 0.0411 & 1596.7 & 25 & 1340 & 12 & 1442 & 10 & 84 & 11 & $1,373,742$ & 134,877 & 23 & $5,764,943$ & 0.0001 & Inc & \\
\hline 13 & 0.0981 & 0.0016 & 0.2241 & 0.0025 & 3.0302 & 0.0492 & 1588.7 & 31 & 1303 & 13 & 1415 & 12 & 82 & 29 & $2,621,084$ & 257,454 & 521 & $12,092,409$ & 0.0001 & Inc & \\
\hline 14 & 0.0978 & 0.0017 & 0.2462 & 0.0029 & 3.3195 & 0.0566 & 1582.5 & 32 & 1419 & 15 & 148 & 13 & 90 & 34 & 88,275 & 8696 & 9 & 377,705 & 0.0039 & Exc & $\mathrm{a}$ \\
\hline 15 & 0.0993 & 0.0019 & 0.2804 & 0.0035 & 3.8364 & 0.0724 & 1610.5 & 35 & 1594 & 18 & 1600 & 15 & 99 & 19 & 397,112 & 40,162 & 164 & $1,571,576$ & 0.0005 & Inc & 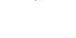 \\
\hline 16 & 0.0972 & 0.0018 & 0.2600 & 0.0031 & 3.4847 & 0.0620 & 1571.8 & 34 & 1490 & 16 & 1524 & 14 & 95 & 0 & 150,286 & 14,739 & 45 & 614,635 & 0.0000 & Inc & \\
\hline 17 & 0.0991 & 0.0012 & 0.2471 & 0.0023 & 3.3736 & 0.0385 & 1606.3 & 23 & 1423 & 12 & 1498 & 9 & 89 & 53 & $1,036,758$ & 102,851 & 278 & $3,725,470$ & 0.0005 & Inc & \\
\hline 18 & 0.0983 & 0.0017 & 0.2288 & 0.0026 & 3.1005 & 0.0525 & 1592.3 & 32 & 1328 & 14 & 1433 & 13 & 83 & 1 & $2,603,502$ & 256,726 & 61 & $11,838,008$ & 0.0000 & Inc & \\
\hline 19 & 0.0991 & 0.0013 & 0.2094 & 0.0019 & 2.8612 & 0.0332 & 1607.3 & 24 & 1226 & 10 & 1372 & 9 & 76 & 24 & 990,138 & 98,206 & 53 & $4,177,061$ & 0.0002 & Inc & \\
\hline 20 & 0.0986 & 0.0019 & 0.2421 & 0.0030 & 3.2912 & 0.0623 & 1598.1 & 36 & 1398 & 15 & 1479 & 15 & 87 & 60 & $2,274,552$ & 225,982 & 215 & $10,140,658$ & 0.0003 & Inc & \\
\hline 21 & 0.0993 & 0.0014 & 0.2208 & 0.0022 & 3.0221 & 0.0403 & 1610.6 & 26 & 1286 & 12 & 1413 & 10 & 80 & 25 & $1,242,696$ & 123,093 & 74 & $5,310,694$ & 0.0002 & Inc & \\
\hline 22 & 0.0997 & 0.0013 & 0.2064 & 0.0019 & 2.8359 & 0.0339 & 1618.0 & 24 & 1210 & 10 & 1365 & 9 & 75 & 11 & 686,332 & 68,503 & 27 & $2,888,126$ & 0.0002 & Inc & \\
\hline 23 & 0.1004 & 0.0013 & 0.2224 & 0.0020 & 3.0771 & 0.0370 & 1630.6 & 25 & 1295 & 11 & 1427 & 9 & 79 & 16 & $1,329,609$ & 133,557 & 307 & $5,178,292$ & 0.0001 & Inc & \\
\hline 24 & 0.0995 & 0.0013 & 0.2182 & 0.0020 & 2.9923 & 0.0361 & 1614.6 & 24 & 1272 & 11 & 1406 & 9 & 79 & 14 & $1,841,114$ & 183,403 & 135 & $7,458,065$ & 0.0001 & Inc & \\
\hline 25 & 0.0980 & 0.0013 & 0.2217 & 0.0021 & 2.9930 & 0.0365 & 1585.4 & 25 & 1291 & 11 & 1406 & 9 & 81 & 15 & $1,573,534$ & 154,374 & 80 & $6,220,637$ & 0.0001 & Inc & \\
\hline 26 & 0.0990 & 0.0017 & 0.2254 & 0.0025 & 3.0762 & 0.0500 & 1605.1 & 31 & 1311 & 13 & 1427 & 12 & 82 & 9 & $1,181,199$ & 116,969 & 18 & $5,122,869$ & 0.0001 & Inc & \\
\hline 27 & 0.1029 & 0.0014 & 0.2496 & 0.0024 & 3.5399 & 0.0445 & 1676.4 & 25 & 1437 & 12 & 1536 & 10 & 86 & 13 & 209,668 & 21,612 & 11 & 745,289 & 0.0006 & Exc & $\mathrm{b}$ \\
\hline 28 & 0.0993 & 0.0023 & 0.2475 & 0.0032 & 3.3886 & 0.0761 & 1610.9 & 42 & 1426 & 17 & 1502 & 18 & 89 & 19 & $1,609,400$ & 162,276 & 10 & $7,066,943$ & 0.0001 & Inc & \\
\hline 29 & 0.1002 & 0.0022 & 0.2555 & 0.0032 & 3.5266 & 0.0743 & 1626.8 & 40 & 1467 & 17 & 1533 & 17 & 90 & 47 & $1,071,385$ & 108,255 & 60 & $4,488,290$ & 0.0004 & Inc & \\
\hline 30 & 0.0988 & 0.0025 & 0.2525 & 0.0035 & 3.4378 & 0.0833 & 1601.6 & 46 & 1451 & 18 & 1513 & 19 & 91 & 14 & $1,946,683$ & 195,519 & 68 & $8,610,834$ & 0.0001 & Inc & \\
\hline 31 & 0.1006 & 0.0012 & 0.2438 & 0.0022 & 3.3800 & 0.0359 & 1634.5 & 22 & 1407 & 11 & 1500 & 8 & 86 & 32 & 562,421 & 56,764 & 322 & $2,025,558$ & 0.0006 & Inc & \\
\hline 32 & 0.0989 & 0.0012 & 0.2367 & 0.0022 & 3.2255 & 0.0342 & 1602.5 & 22 & 1370 & 11 & 1463 & 8 & 85 & 4 & 960,716 & 95,346 & 13 & $3,570,458$ & 0.0000 & Inc & \\
\hline 33 & 0.0981 & 0.0012 & 0.2273 & 0.0021 & 3.0728 & 0.0328 & 1587.9 & 22 & 1320 & 11 & 1426 & 8 & 83 & 18 & 759,636 & 74,830 & 179 & $2,937,270$ & 0.0002 & Inc & \\
\hline 34 & 0.0988 & 0.0014 & 0.2231 & 0.0023 & 3.0405 & 0.0422 & 1602.4 & 27 & 1298 & 12 & 1418 & 11 & 81 & 10 & 444,428 & 44,200 & 46 & $1,912,461$ & 0.0002 & Inc & \\
\hline 35 & 0.1158 & 0.0014 & 0.2723 & 0.0026 & 4.3452 & 0.0491 & 1891.7 & 22 & 1552 & 13 & 1702 & 9 & 82 & 104 & 87,658 & 10,202 & 5406 & 286,152 & 0.0102 & Exc & c \\
\hline 36 & 0.1207 & 0.0015 & 0.3719 & 0.0035 & 6.1896 & 0.0706 & 1966.9 & 22 & 2038 & 16 & 2003 & 10 & 104 & 202 & 82,870 & 10,054 & 8583 & 199,203 & 0.0201 & Exc & c \\
\hline 37 & 0.0997 & 0.0014 & 0.3164 & 0.0027 & 4.3477 & 0.0536 & 1618.2 & 26 & 1772 & 13 & 1703 & 10 & 109 & 39 & 360,353 & 36,098 & 1705 & 888,545 & 0.0011 & Exc & c \\
\hline 38 & 0.0976 & 0.0015 & 0.2263 & 0.0025 & 3.0452 & 0.0461 & 1579.1 & 28 & 1315 & 13 & 1419 & 12 & 83 & 42 & $2,915,401$ & 287,873 & 68 & $13,379,398$ & 0.0001 & Inc & \\
\hline 39 & 0.0973 & 0.0015 & 0.2418 & 0.0027 & 3.2435 & 0.0495 & 1572.9 & 29 & 1396 & 14 & 1468 & 12 & 89 & 24 & $1,084,105$ & 106,636 & 81 & $4,645,543$ & 0.0002 & Inc & \\
\hline 40 & 0.0970 & 0.0016 & 0.2333 & 0.0027 & 3.1184 & 0.0522 & 1566.7 & 31 & 1352 & 14 & 143 & 13 & 86 & 20 & $1,166,921$ & 115,101 & 97 & $5,317,064$ & 0.0002 & Inc & \\
\hline 41 & 0.0977 & 0.0015 & 0.2303 & 0.0026 & 3.1028 & 0.0479 & 1581.0 & 29 & 1336 & 14 & 14 & 12 & 85 & 65 & $3,206,861$ & 317,610 & 271 & $14,493,242$ & 0.0002 & Inc & \\
\hline 42 & 0.0985 & 0.0016 & 0.2251 & 0.0026 & 3.0580 & 0.0485 & 1596.1 & 30 & 1309 & 13 & 142 & 12 & 82 & 25 & $3,258,201$ & 325,873 & 16 & $15,085,051$ & 0.0001 & Inc & \\
\hline 43 & 0.0979 & 0.0016 & 0.2355 & 0.0027 & 3.1780 & 0.0524 & 1584.1 & 31 & 1363 & 14 & 1452 & 13 & 86 & 34 & $2,438,001$ & 242,741 & 219 & $10,914,086$ & 0.0001 & Inc & \\
\hline 44 & 0.0984 & 0.0018 & 0.2337 & 0.0027 & 3.1695 & 0.0562 & 1593.6 & 34 & 1354 & 14 & 1450 & 14 & 85 & 15 & 993,572 & 99,903 & 83 & $4,366,896$ & 0.0002 & Inc & \\
\hline 45 & 0.0976 & 0.0019 & 0.2602 & 0.0032 & 3.5019 & 0.0669 & 1579.2 & 36 & 1491 & 17 & 1528 & 15 & 94 & 15 & $1,812,700$ & 182,136 & 143 & $7,664,977$ & 0.0001 & Inc & \\
\hline 46 & 0.1001 & 0.0013 & 0.2242 & 0.0021 & 3.0947 & 0.0365 & 1626.4 & 24 & 1304 & 11 & 143 & 9 & 80 & 29 & 302,131 & 30,514 & 202 & $1,200,461$ & 0.0010 & Inc & \\
\hline 47 & 0.0993 & 0.0021 & 0.2756 & 0.0036 & 3.77 & 0.0789 & 161 & 39 & 15 & 18 & 1 & 17 & 97 & 34 & 1,198 & 122 & 61 & 4,8 & 0.0003 & Inc & \\
\hline 48 & 0.0982 & 0.0013 & 0.2188 & 0.0020 & 2.9622 & 0.0352 & 1589.9 & 24 & 12 & 11 & 13 & 9 & 80 & 23 & $1,668,670$ & 165,554 & 22 & $6,676,239$ & 0.0001 & Inc & \\
\hline 49 & 0.0990 & 0.0013 & 0.2173 & 0.0020 & 2.9663 & 0.0355 & 1605.7 & 24 & 1268 & 11 & 1399 & 9 & 79 & 7 & $1,855,607$ & 185,626 & 43 & $7,550,038$ & 0.0000 & Inc & \\
\hline 50 & 0.0989 & 0.0013 & 0.2221 & 0.0021 & 3.0278 & 0.0366 & 1603.2 & 24 & 1293 & 11 & 1415 & 9 & 81 & 20 & $1,805,412$ & 180,401 & 10 & $7,238,337$ & 0.0001 & Inc & \\
\hline
\end{tabular}


Table 4. Cont.

\begin{tabular}{|c|c|c|c|c|c|c|c|c|c|c|c|c|c|c|c|c|c|c|c|c|c|}
\hline $\begin{array}{l}\text { Spot } \\
\text { No. }\end{array}$ & ${ }^{207} \mathrm{~Pb} /{ }^{206} \mathrm{~Pb}$ & $1 \sigma$ & ${ }^{206} \mathrm{~Pb} /{ }^{238} \mathrm{U}$ & $1 \sigma$ & ${ }^{207} \mathrm{~Pb} /{ }^{235} \mathrm{U}$ & $1 \sigma$ & ${ }^{207} \mathrm{~Pb} / 206 \mathrm{~Pb}$ & $1 \sigma$ & ${ }^{206} \mathrm{~Pb} /{ }^{238} \mathrm{U}$ & $1 \sigma$ & ${ }^{207} \mathrm{~Pb} /{ }^{235} \mathrm{U}$ & $1 \sigma$ & $\begin{array}{c}\text { Concordancy } \\
(\%)\end{array}$ & ${ }^{204} \mathrm{~Pb}$ & ${ }^{206} \mathrm{~Pb}$ & ${ }^{207} \mathrm{~Pb}$ & ${ }^{208} \mathrm{~Pb}$ & ${ }^{238} \mathrm{U}$ & ${ }^{204} \mathrm{~Pb} /{ }^{207} \mathrm{~Pb}$ & Inc/Exc & Notes \\
\hline 51 & 0.0989 & 0.0013 & 0.2260 & 0.0021 & 3.0802 & 0.0376 & 1602.9 & 25 & 1313 & 11 & 1428 & 9 & 82 & 22 & $1,366,690$ & 136,630 & 12 & $5,394,476$ & 0.0002 & Inc & \\
\hline 52 & 0.0981 & 0.0013 & 0.2263 & 0.0021 & 3.0593 & 0.0377 & 1587.8 & 25 & 1315 & 11 & 1423 & 9 & 83 & 13 & $1,403,969$ & 139,309 & 31 & $5,542,698$ & 0.0001 & Inc & \\
\hline 53 & 0.0987 & 0.0013 & 0.2182 & 0.0021 & 2.9691 & 0.0371 & 1599.5 & 25 & 1272 & 11 & 1400 & 9 & 80 & 22 & $1,619,038$ & 161,606 & 44 & $6,603,413$ & 0.0001 & Inc & \\
\hline 54 & 0.0982 & 0.0016 & 0.2343 & 0.0026 & 3.1730 & 0.0511 & 1590.4 & 31 & 1357 & 14 & 1451 & 12 & 85 & 11 & $1,610,758$ & 161,574 & 29 & $6,854,733$ & 0.0001 & Inc & \\
\hline 55 & 0.0976 & 0.0014 & 0.2250 & 0.0022 & 3.0270 & 0.0387 & 1578.4 & 26 & 1308 & 11 & 1415 & 10 & 83 & 18 & 457,802 & 45,280 & 177 & $1,824,297$ & 0.0004 & Inc & \\
\hline 56 & 0.0970 & 0.0022 & 0.2539 & 0.0034 & 3.3953 & 0.0773 & 1567.3 & 43 & 1459 & 17 & 1503 & 18 & 93 & 36 & $4,452,099$ & 443,120 & 34 & $19,586,568$ & 0.0001 & Inc & \\
\hline 57 & 0.0972 & 0.0014 & 0.2060 & 0.0020 & 2.7602 & 0.0360 & 1571.2 & 26 & 1207 & 11 & 1345 & 10 & 77 & 14 & 959,312 & 94,476 & 14 & $4,158,679$ & 0.0001 & Inc & \\
\hline 58 & 0.0976 & 0.0014 & 0.2142 & 0.0021 & 2.8808 & 0.0380 & 1577.8 & 26 & 1251 & 11 & 1377 & 10 & 79 & 20 & $1,450,919$ & 143,445 & 34 & $6,049,981$ & 0.0001 & Inc & \\
\hline 59 & 0.0986 & 0.0014 & 0.2261 & 0.0022 & 3.0741 & 0.0411 & 1598.2 & 27 & 1314 & 12 & 1426 & 10 & 82 & 15 & 571,895 & 57,181 & 82 & $2,260,135$ & 0.0003 & Inc & \\
\hline 60 & 0.0967 & 0.0018 & 0.2356 & 0.0027 & 3.1402 & 0.0582 & 1560.9 & 35 & 1364 & 14 & 1443 & 14 & 87 & 19 & 956,335 & 95,287 & 35 & $4,086,355$ & 0.0002 & Inc & \\
\hline 61 & 0.1052 & 0.0015 & 0.2473 & 0.0027 & 3.5852 & 0.0494 & 1718.0 & 26 & 1425 & 14 & 1546 & 11 & 83 & 16 & 254,148 & 26,922 & 29 & $1,046,269$ & 0.0006 & Exc & $\mathrm{b}$ \\
\hline 62 & 0.1041 & 0.0012 & 0.2105 & 0.0020 & 3.0188 & 0.0328 & 1697.5 & 22 & 1232 & 10 & 1412 & 8 & 73 & 6 & 184,596 & 19,297 & 61 & 782,648 & 0.0003 & Exc & d \\
\hline 63 & 0.0990 & 0.0016 & 0.2346 & 0.0027 & 3.1989 & 0.0513 & 1605.2 & 30 & 1358 & 14 & 1457 & 12 & 85 & 4 & $1,925,329$ & 192,516 & 55 & $8,914,925$ & 0.0000 & Inc & \\
\hline 64 & 0.0989 & 0.0017 & 0.2147 & 0.0025 & 2.9234 & 0.0484 & 1602.8 & 31 & 1254 & 13 & 1388 & 13 & 78 & 9 & 792,921 & 79,559 & 35 & $3,850,466$ & 0.0001 & Inc & \\
\hline 65 & 0.0976 & 0.0013 & 0.2318 & 0.0025 & 3.1167 & 0.0421 & 1579.0 & 25 & 1344 & 13 & 1437 & 10 & 85 & 3 & $1,765,155$ & 173,074 & 216 & $7,785,311$ & 0.0000 & Inc & \\
\hline 66 & 0.0997 & 0.0014 & 0.2364 & 0.0026 & 3.2466 & 0.0451 & 1618.1 & 26 & 1368 & 13 & 1468 & 11 & 85 & 101 & $1,098,693$ & 110,264 & 287 & $4,758,180$ & 0.0009 & Exc & c \\
\hline 67 & 0.0980 & 0.0014 & 0.2388 & 0.0025 & 3.2225 & 0.0437 & 1585.5 & 26 & 1380 & 13 & 1463 & 11 & 87 & 3 & 933,955 & 92,136 & 299 & $3,898,835$ & 0.0000 & Inc & \\
\hline 68 & 0.1143 & 0.0015 & 0.2331 & 0.0022 & 3.6724 & 0.0423 & 1868.7 & 23 & 1351 & 11 & 1565 & 9 & 72 & 17 & 79,178 & 9127 & 999 & 295,491 & 0.0019 & Exc & a \\
\hline 69 & 0.0985 & 0.0012 & 0.2054 & 0.0019 & 2.7877 & 0.0299 & 1595.0 & 22 & 1204 & 10 & 1352 & 8 & 76 & 22 & $1,439,121$ & 142,793 & 43 & $6,191,064$ & 0.0002 & Inc & a \\
\hline 70 & 0.0973 & 0.0012 & 0.2362 & 0.0023 & 3.1664 & 0.0380 & 1572.5 & 23 & 1367 & 12 & 1449 & 9 & 87 & 12 & $1,175,148$ & 115,582 & 47 & $4,740,360$ & 0.0001 & Inc & \\
\hline 71 & 0.0980 & 0.0013 & 0.2282 & 0.0023 & 3.0837 & 0.0392 & 1587.2 & 25 & 1325 & 12 & 1429 & 10 & 83 & 10 & $1,054,676$ & 104,286 & 9 & $4,487,131$ & 0.0001 & Inc & \\
\hline 72 & 0.0986 & 0.0017 & 0.2622 & 0.0031 & 3.5627 & 0.0600 & 1598.5 & 31 & 1501 & 16 & 1541 & 13 & 94 & 19 & 642,983 & 63,740 & 115 & $2,647,484$ & 0.0003 & Inc & \\
\hline 73 & 0.0996 & 0.0014 & 0.2243 & 0.0021 & 3.0788 & 0.0396 & 1616.1 & 26 & 1305 & 11 & 1427 & 10 & 81 & 23 & 29,009 & 2923 & 59 & 110,735 & 0.0079 & Exc & a \\
\hline 74 & 0.0993 & 0.0022 & 0.1845 & 0.0022 & 2.5247 & 0.0509 & 1610.1 & 40 & 1092 & 12 & 1279 & 15 & 68 & 22 & 4875 & 488 & 19 & 22,920 & 0.0451 & Exc & $\mathrm{a}$ \\
\hline 75 & 0.0988 & 0.0016 & 0.2190 & 0.0025 & 2.9815 & 0.0482 & 1601.3 & 30 & 1277 & 13 & 1403 & 12 & 80 & 8 & $1,979,677$ & 196,785 & 49 & $9,503,796$ & 0.0000 & Inc & \\
\hline 76 & 0.0973 & 0.0012 & 0.1995 & 0.0018 & 2.6761 & 0.0298 & 1573.2 & 23 & 1173 & 10 & 1322 & 8 & 75 & 21 & 399,834 & 39,344 & 144 & $1,748,439$ & 0.0005 & Inc & \\
\hline 77 & 0.0987 & 0.0014 & 0.2291 & 0.0024 & 3.1165 & 0.0439 & 1599.4 & 27 & 1330 & 13 & 1437 & 11 & 83 & 0 & $1,078,987$ & 107,212 & 210 & $4,628,883$ & 0.0000 & Inc & \\
\hline 78 & 0.1001 & 0.0017 & 0.2259 & 0.0026 & 3.1157 & 0.0518 & 1626.2 & 31 & 1313 & 14 & 1437 & 13 & 81 & 16 & $2,587,938$ & 260,008 & 462 & $12,235,322$ & 0.0001 & Inc & \\
\hline 79 & 0.0981 & 0.0012 & 0.2321 & 0.0021 & 3.1378 & 0.0353 & 1587.7 & 23 & 1346 & 11 & 1442 & 9 & 85 & 14 & 620,074 & 61,490 & 192 & $2,342,069$ & 0.0002 & Inc & \\
\hline 80 & 0.0992 & 0.0012 & 0.2509 & 0.0023 & 3.4306 & 0.0390 & 1608.8 & 23 & 1443 & 12 & 1511 & 9 & 90 & 22 & 455,461 & 45,655 & 30 & $1,607,845$ & 0.0005 & Inc & \\
\hline 81 & 0.0990 & 0.0013 & 0.2408 & 0.0022 & 3.2874 & 0.0374 & 1605.6 & 23 & 1391 & 11 & 1478 & 9 & 87 & 15 & 834,712 & 83,678 & 19 & $3,004,981$ & 0.0002 & Inc & \\
\hline 82 & 0.0979 & 0.0012 & 0.2179 & 0.0020 & 2.9404 & 0.0336 & 1583.9 & 23 & 1271 & 11 & 1392 & 9 & 80 & 20 & 886,685 & 87,861 & 31 & $3,560,567$ & 0.0002 & Inc & \\
\hline 83 & 0.1012 & 0.0014 & 0.2326 & 0.0023 & 3.2455 & 0.0406 & 1646.0 & 25 & 1348 & 12 & 1468 & 10 & 82 & 0 & 476,332 & 48,675 & 12 & $1,865,476$ & 0.0000 & Inc & \\
\hline 84 & 0.0981 & 0.0013 & 0.2461 & 0.0023 & 3.3268 & 0.0389 & 1587.5 & 24 & 1418 & 12 & 14 & 9 & 89 & 24 & 303,591 & 30,129 & 12 & $1,090,386$ & 0.0008 & Inc & \\
\hline 85 & 0.0976 & 0.0014 & 0.2102 & 0.0020 & 2.8297 & 0.0361 & 1579.3 & 26 & 1230 & 11 & 136 & 10 & 78 & 14 & 43,652 & 43 & 7 & 18 & 0.0032 & Exc & a \\
\hline 86 & 0.0971 & 0.0014 & 0.2156 & 0.0022 & 2.8871 & 0.0384 & 1569.6 & 26 & 1259 & 11 & 13 & 10 & 80 & 20 & 856,148 & 83,793 & 50 & $3,731,805$ & 0.0002 & Inc & \\
\hline 87 & 0.0979 & 0.0021 & 0.2542 & 0.0033 & 3.4275 & 0.0721 & 1584.5 & 39 & 1460 & 17 & 1511 & 17 & 92 & 23 & $1,736,866$ & 171,672 & 17 & $7,714,529$ & 0.0001 & Inc & \\
\hline 88 & 0.0986 & 0.0020 & 0.2328 & 0.0029 & 3.1632 & 0.0640 & 1598.5 & 38 & 1349 & 15 & 1448 & 16 & 84 & 21 & $2,302,525$ & 227,831 & 27 & $10,881,509$ & 0.0001 & Inc & \\
\hline 89 & 0.0994 & 0.0022 & 0.2475 & 0.0032 & 3.3875 & 0.0744 & 1612.1 & 41 & 1425 & 16 & 1502 & 17 & 88 & 5 & $2,176,639$ & 214,810 & 625 & $9,634,952$ & 0.0000 & Inc & \\
\hline 90 & 0.0966 & 0.0022 & 0.2829 & 0.0038 & 3.7626 & 0.0857 & 1558.5 & 43 & 1606 & 19 & 1585 & 18 & 103 & 22 & $1,157,825$ & 112,175 & 4 & $4,575,889$ & 0.0002 & Inc & \\
\hline 91 & 0.0987 & 0.0023 & 0.2744 & 0.0037 & 3.7291 & 0.0859 & 1599.1 & 43 & 15 & 19 & 15 & 18 & 98 & 35 & 935,888 & 93,406 & 143 & $3,881,266$ & 0.0004 & Inc & \\
\hline 92 & 0.0993 & 0.0 & 0.2 & 0.0036 & 3.6 & 0.0 & 161 & 43 & 15 & 18 & 15 & 18 & 94 & 21 & & & 108 & & 0.0008 & Inc & \\
\hline 93 & 0.0997 & 0.0026 & 0.2754 & 0.0039 & 3.7788 & 0.0965 & 1617.5 & 48 & 1568 & 20 & 158 & 21 & 97 & 11 & $1,194,297$ & 118,707 & 124 & $4,905,337$ & 0.0001 & Inc & \\
\hline 94 & 0.0974 & 0.0021 & 0.2478 & 0.0032 & 3.3247 & 0.0702 & 1574.5 & 40 & 1427 & 16 & 1487 & 16 & 91 & 27 & 303,011 & 29,789 & 168 & $1,328,016$ & 0.0009 & Inc & \\
\hline 95 & 0.1971 & 0.0028 & 0.4371 & 0.0042 & 11.8798 & 0.1518 & 2802.5 & 23 & 2338 & 19 & 2595 & 12 & 83 & 548 & 73,261 & 14,660 & 30,042 & 147,870 & 0.0374 & Exc & c \\
\hline
\end{tabular}

Notes: a: High ${ }^{204 / 207}$ ratio; b: Low ${ }^{207} \mathrm{~Pb}$ cts.; c: High ${ }^{204} \mathrm{~Pb}$ cts.; d: Low ${ }^{238} \mathrm{U}$ cts. 
Sample PH-93 was dated at an upper intercept age of $1604 \pm 11$ Ma with an anchored lower intercept of $0 \pm 40 \mathrm{Ma}$, using a 27- $\mu \mathrm{m}$ spot size and a 12-ppb mixed solution. Sample PH-93 (Figure 5) plots very close to concordia, but displays evidence of slight recent $\mathrm{Pb}$ loss like OD10-4. Sample PH-93 was also dated using GJ-1 Zircon as the primary standard, giving an age of 1597.2 $\pm 6.1 \mathrm{Ma}$ (Figure 5) utilizing 82 of a total of 95 points (Table 4). Although the error is small, the range of discordance is large when compared to the solution analysis, in which spots plot close to or on concordia (Figure 4). The larger spread in PH93 (b) could, however, be due to the larger number of analyses.

Due to its much lower $U$ concentration (average $U$ is 6 ppm), sample CUR-002 was analyzed using a $60-\mu \mathrm{m}$ spot size and a 1-ppb mixed solution. The sample contains extremely high ${ }^{204} \mathrm{~Pb}$, exemplified by how far the points plot from the lower intercept on the Tera-Wasserburg diagram. The most robust data points, marked in red, have been included in the age regression (Figure 6, Table 5), which produced a lower intercept date of $1707 \pm 39 \mathrm{Ma}$ from 40 out of 60 points. When all data are plotted, an age of $1711 \pm 120 \mathrm{Ma}$ is produced, excluding two points that gave ${ }^{204} \mathrm{~Pb}$ counts which were too high $(>700)$, and one with measured age ratios which were unrealistically old (point 3 ) and therefore are not included in either date. Despite high levels of ${ }^{204} \mathrm{~Pb}$, the fitted regression line (from ISOPLOT using the algorithm of York, 1969 [18]) produces a low mean square weighted deviation (MSWD) and relatively low error, demonstrating how even samples of very low $\mathrm{U}$ and high common $\mathrm{Pb}$ can still give geologically meaningful ages.

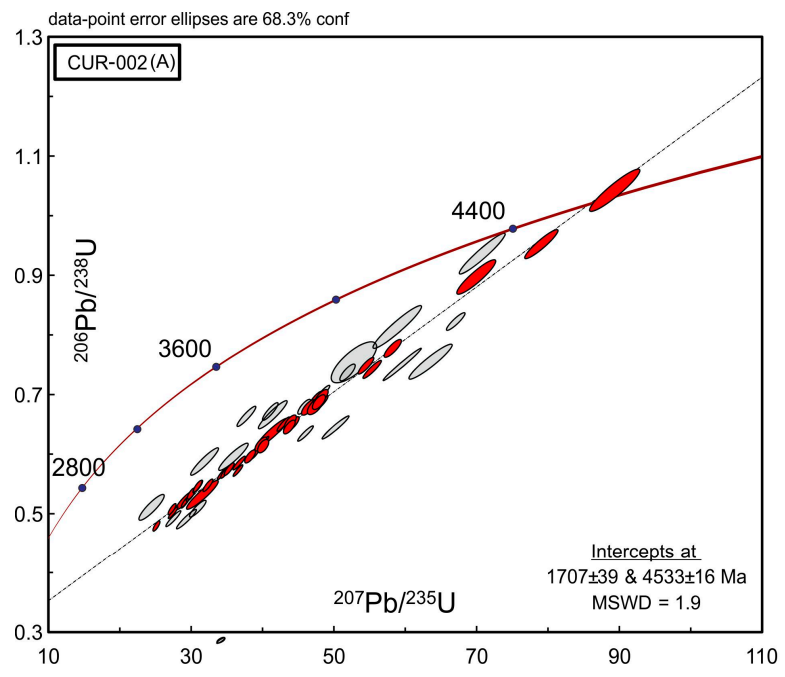

(a)

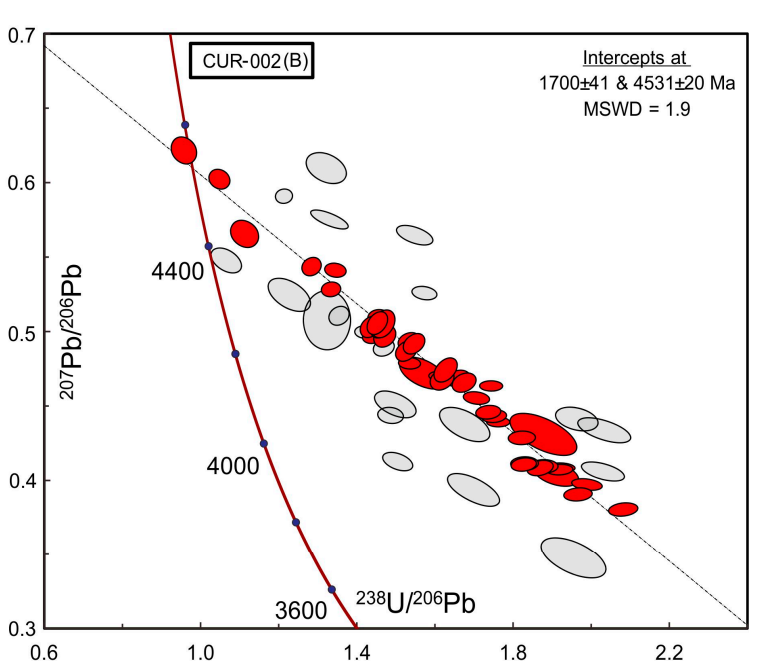

(b)

Figure 6. Conventional concordia plot (a) and Tera-Wasserburg diagram (b) for the low-U sample (CUR-002; Carajás, Brazil). The more robust data points marked in red are included in the age regression. Data given in Table 5 as "included" are plotted in red, "excluded" as grey. 
Table 5. Geochronological data for zoned hematite (sample CUR-002) using mixed-solution method.

\begin{tabular}{|c|c|c|c|c|c|c|c|c|c|c|c|c|c|c|c|c|c|c|c|c|c|}
\hline $\begin{array}{l}\text { Spot } \\
\text { No. }\end{array}$ & ${ }^{207} \mathrm{~Pb} /{ }^{206} \mathrm{~Pb}$ & $1 \sigma$ & ${ }^{206} \mathrm{~Pb} /{ }^{238} \mathrm{U}$ & $1 \sigma$ & ${ }^{207} \mathrm{~Pb} /{ }^{235} \mathrm{U}$ & $1 \sigma$ & ${ }^{207} \mathrm{~Pb} /{ }^{206} \mathrm{~Pb}$ & $1 \sigma$ & ${ }^{206} \mathrm{~Pb} /{ }^{238} \mathrm{U}$ & $1 \sigma$ & ${ }^{207} \mathrm{~Pb} /{ }^{235} \mathrm{U}$ & $1 \sigma$ & $\begin{array}{c}\text { Concordancy } \\
(\%)\end{array}$ & ${ }^{204} \mathrm{~Pb}$ & ${ }^{206} \mathrm{~Pb}$ & ${ }^{207} \mathrm{~Pb}$ & ${ }^{208} \mathrm{~Pb}$ & ${ }^{238} \mathrm{U}$ & ${ }^{204} \mathrm{~Pb} /{ }^{207} \mathrm{~Pb}$ & Inc/Exc & Notes \\
\hline 1 & 0.5079 & 0.0237 & 0.7545 & 0.0227 & 52.8353 & 2.0992 & 4264 & 67 & 3624 & 88 & 4047 & 45 & 90 & 15 & 695 & 349 & 889 & 1225 & 0.04298 & Exc & \\
\hline 2 & 0.5755 & 0.0197 & 0.7510 & 0.0179 & 59.5928 & 1.7618 & 4447 & 49 & 3611 & 70 & 4167 & 34 & 87 & 30 & 687 & 391 & 949 & 1217 & 0.07673 & Exc & \\
\hline 3 & 0.7995 & 0.0107 & 2.7586 & 0.0389 & 304.0886 & 4.4477 & 4921 & 19 & 8535 & 74 & 5809 & 17 & 147 & 265 & 5445 & 4307 & 10,609 & 2625 & 0.06153 & Exc & $\mathrm{a}$ \\
\hline 4 & 0.4339 & 0.0162 & 0.4913 & 0.0108 & 29.3881 & 0.9197 & 4031 & 55 & 2576 & 49 & 3467 & 35 & 74 & 30 & 804 & 345 & 1025 & 2177 & 0.08696 & Exc & \\
\hline 5 & 0.3478 & 0.0198 & 0.5110 & 0.0143 & 24.5040 & 1.1702 & 3697 & 84 & 2661 & 64 & 3289 & 53 & 81 & 19 & 373 & 128 & 368 & 972 & 0.14844 & Exc & \\
\hline 6 & 0.5249 & 0.0230 & 0.8138 & 0.0237 & 58.8979 & 2.2419 & 4313 & 63 & 3838 & 89 & 4156 & 44 & 92 & 35 & 369 & 191 & 495 & 604 & 0.18325 & Exc & \\
\hline 7 & 0.5480 & 0.0181 & 0.9369 & 0.0226 & 70.7932 & 2.1173 & 4376 & 48 & 4262 & 80 & 4340 & 34 & 98 & 36 & 778 & 421 & 1083 & 1105 & 0.08551 & Exc & \\
\hline 8 & 0.3938 & 0.0187 & 0.5880 & 0.0152 & 31.9254 & 1.2866 & 3886 & 70 & 2981 & 65 & 3548 & 45 & 84 & 19 & 416 & 162 & 429 & 941 & 0.11728 & Exc & \\
\hline 9 & 0.6101 & 0.0222 & 0.7556 & 0.0194 & 63.5579 & 1.9968 & 4532 & 52 & 3628 & 75 & 4232 & 36 & 86 & 37 & 562 & 339 & 870 & 989 & 0.10914 & Exc & \\
\hline 10 & 0.4062 & 0.0120 & 0.4921 & 0.0088 & 27.5560 & 0.6905 & 3932 & 43 & 2580 & 40 & 3403 & 28 & 76 & 8 & 1217 & 488 & 1594 & 3289 & 0.01639 & Exc & \\
\hline 11 & 0.5650 & 0.0162 & 0.6452 & 0.0128 & 50.2601 & 1.2436 & 4420 & 41 & 3209 & 53 & 3997 & 28 & 80 & 38 & 1003 & 560 & 1500 & 2068 & 0.06786 & Exc & \\
\hline 12 & 0.4724 & 0.0195 & 0.6368 & 0.0160 & 41.4764 & 1.4602 & 4157 & 60 & 3176 & 66 & 3807 & 40 & 83 & 13 & 455 & 212 & 545 & 951 & 0.06132 & Inc & \\
\hline 13 & 0.4312 & 0.0239 & 0.5323 & 0.0160 & 31.6463 & 1.4703 & 4022 & 80 & 2751 & 70 & 3539 & 52 & 78 & 8 & 342 & 146 & 367 & 856 & 0.05479 & Inc & \\
\hline 14 & 0.4377 & 0.0196 & 0.5959 & 0.0151 & 35.9663 & 1.3620 & 4044 & 65 & 3013 & 64 & 3666 & 43 & 82 & 13 & 398 & 172 & 430 & 888 & 0.07558 & Exc & \\
\hline 15 & 0.5415 & 0.0091 & 0.7428 & 0.0100 & 55.4611 & 0.8533 & 4358 & 24 & 3581 & 39 & 4096 & 18 & 87 & 141 & 4477 & 2394 & 5560 & 8013 & 0.05890 & Inc & \\
\hline 16 & 0.5260 & 0.0092 & 0.6350 & 0.0085 & 46.0560 & 0.7213 & 4316 & 25 & 3169 & 35 & 3911 & 18 & 81 & 82 & 2763 & 1435 & 3412 & 5785 & 0.05714 & Exc & \\
\hline 17 & 0.6219 & 0.0175 & 1.0434 & 0.0234 & 89.4817 & 2.3285 & 4560 & 40 & 4607 & 79 & 4574 & 30 & 101 & 31 & 816 & 507 & 1251 & 1028 & 0.06114 & Inc & \\
\hline 18 & 0.6026 & 0.0128 & 0.9530 & 0.0161 & 79.1910 & 1.5412 & 4514 & 30 & 4315 & 57 & 4452 & 22 & 97 & 39 & 1436 & 865 & 2136 & 1982 & 0.04509 & Inc & \\
\hline 19 & 0.5659 & 0.0162 & 0.8979 & 0.0191 & 70.0616 & 1.8009 & 4423 & 41 & 4131 & 69 & 4329 & 30 & 95 & 15 & 953 & 539 & 1332 & 1397 & 0.02783 & Inc & \\
\hline 20 & 0.4046 & 0.0134 & 0.5231 & 0.0103 & 29.1756 & 0.8171 & 3926 & 49 & 2712 & 46 & 3459 & 31 & 78 & 22 & 1246 & 504 & 1187 & 3135 & 0.04365 & Inc & \\
\hline 21 & 0.4512 & 0.0169 & 0.6666 & 0.0156 & 41.4718 & 1.3298 & 4089 & 54 & 3293 & 64 & 3807 & 36 & 86 & 36 & 706 & 318 & 837 & 1393 & 0.11321 & Exc & \\
\hline 22 & 0.4556 & 0.0079 & 0.5857 & 0.0076 & 36.7934 & 0.5699 & 4104 & 26 & 2972 & 33 & 3688 & 18 & 81 & 85 & 3205 & 1458 & 3389 & 7199 & 0.05830 & Inc & \\
\hline 23 & 0.4090 & 0.0059 & 0.5192 & 0.0060 & 29.2778 & 0.3877 & 3942 & 22 & 2696 & 27 & 3463 & 15 & 78 & 113 & 5986 & 2444 & 5153 & 15,168 & 0.04624 & Inc & \\
\hline 24 & 0.4637 & 0.0061 & 0.5731 & 0.0064 & 36.6418 & 0.4497 & 4130 & 19 & 2920 & 28 & 3684 & 14 & 79 & 228 & 8706 & 4031 & 9108 & 19,990 & 0.05656 & Inc & \\
\hline 25 & 0.4704 & 0.0062 & 0.6199 & 0.0070 & 40.2129 & 0.4940 & 4151 & 19 & 3110 & 29 & 3776 & 14 & 82 & 207 & 8683 & 4077 & 9156 & 18,431 & 0.05077 & Inc & \\
\hline 26 & 0.4395 & 0.0057 & 0.5672 & 0.0063 & 34.3697 & 0.4176 & 4050 & 19 & 2896 & 27 & 3621 & 14 & 80 & 185 & 9174 & 4023 & 8902 & 21,285 & 0.04599 & Inc & \\
\hline 27 & 0.3975 & 0.0073 & 0.5024 & 0.0065 & 27.5376 & 0.4435 & 3900 & 27 & 2624 & 29 & 3403 & 18 & 77 & 90 & 4110 & 1630 & 3469 & 10,768 & 0.05521 & Inc & \\
\hline 28 & 0.4998 & 0.0075 & 0.7029 & 0.0086 & 48.4418 & 0.6644 & 4241 & 22 & 3431 & 34 & 3961 & 16 & 87 & 110 & 4804 & 2395 & 5666 & 8995 & 0.04593 & Exc & \\
\hline 29 & 0.4129 & 0.0109 & 0.6638 & 0.0112 & 37.7835 & 0.8694 & 3957 & 39 & 3282 & 46 & 3714 & 26 & 88 & 29 & 1052 & 433 & 1124 & 2086 & 0.06697 & Exc & \\
\hline 30 & 0.4790 & 0.0071 & 0.6511 & 0.0079 & 42.9972 & 0.5849 & 4178 & 22 & 3232 & 32 & 3842 & 15 & 84 & 140 & 4823 & 2303 & 5181 & 9752 & 0.06079 & Inc & \\
\hline 31 & 0.5911 & 0.0081 & 0.8230 & 0.0098 & 67.0843 & 0.8588 & 4486 & 20 & 3871 & 37 & 4286 & 15 & 90 & 244 & 6976 & 4110 & 9492 & 11,159 & 0.05937 & Exc & \\
\hline 32 & 0.4414 & 0.0129 & 0.5088 & 0.0093 & 30.9697 & 0.7696 & 4056 & 43 & 2652 & 42 & 3518 & 28 & 75 & 36 & 1206 & 530 & 1572 & 3121 & 0.06792 & Exc & \\
\hline 33 & 0.4970 & 0.0064 & 0.6938 & 0.0082 & 47.5389 & 0.6026 & 4232 & 19 & 3397 & 33 & 3942 & 14 & 86 & 257 & 9016 & 4565 & 10,418 & 18,473 & 0.05630 & Inc & \\
\hline 34 & 0.5287 & 0.0072 & 0.7490 & 0.0092 & 54.5962 & 0.7268 & 4323 & 20 & 3604 & 36 & 4080 & 15 & 88 & 174 & 7320 & 3942 & 9031 & 13,891 & 0.04414 & Inc & \\
\hline 35 & 0.5094 & 0.0085 & 0.6856 & 0.0091 & 48.1500 & 0.7354 & 4269 & 24 & 3366 & 37 & 3955 & 17 & 85 & 36 & 3437 & 1783 & 4112 & 7126 & 0.02019 & Inc & \\
\hline 36 & 0.4075 & 0.0052 & 0.5208 & 0.0061 & 29.2629 & 0.3683 & 3937 & 19 & 2703 & 27 & 3462 & 14 & 78 & 259 & 11,938 & 4954 & 10,495 & 32,573 & 0.05228 & Inc & \\
\hline 37 & 0.4097 & 0.0065 & 0.5319 & 0.0068 & 30.0497 & 0.4415 & 3945 & 23 & 2750 & 30 & 3488 & 17 & 79 & 115 & 4992 & 2082 & 4365 & 13,336 & 0.05524 & Inc & \\
\hline 38 & 0.4434 & 0.0059 & 0.5706 & 0.0069 & 34.8889 & 0.4528 & 4063 & 20 & 2910 & 30 & 3636 & 15 & 80 & 248 & 10,188 & 4598 & 10,140 & 25,363 & 0.05394 & Inc & \\
\hline 39 & 0.4438 & 0.0086 & 0.6720 & 0.0098 & 41.1226 & 0.7212 & 4065 & 28 & 3313 & 40 & 3798 & 20 & 87 & 72 & 3388 & 1530 & 3761 & 7163 & 0.04706 & Exc & \\
\hline 40 & 0.3807 & 0.0051 & 0.4800 & 0.0057 & 25.1978 & 0.3247 & 3835 & 20 & 2527 & 26 & 3316 & 14 & 76 & 208 & 10,362 & 4014 & 8264 & 30,661 & 0.05182 & Inc & \\
\hline 41 & 0.5439 & 0.0077 & 0.7781 & 0.0099 & 58.3534 & 0.7965 & 4365 & 21 & 3710 & 38 & 4146 & 16 & 89 & 211 & 6461 & 3575 & 8414 & 11,794 & 0.05902 & Inc & \\
\hline 42 & 0.4461 & 0.0061 & 0.5755 & 0.0070 & 35.3955 & 0.4646 & 4072 & 20 & 2930 & 30 & 3650 & 15 & 80 & 215 & 9036 & 4100 & 9398 & 22,298 & 0.05244 & Inc & \\
\hline 43 & 0.3909 & 0.0055 & 0.5083 & 0.0062 & 27.3917 & 0.3676 & 3874 & 21 & 2649 & 27 & 3398 & 15 & 78 & 224 & 96 & 3832 & 8136 & 26,937 & 0.05846 & Inc & \\
\hline 44 & 0.4289 & 0.0064 & 0.5484 & 0.0069 & 32.4251 & 0.4558 & 4014 & 22 & 2818 & 30 & 3563 & 16 & 79 & 161 & 6409 & 2795 & 6425 & 16,595 & 0.05760 & Inc & \\
\hline 45 & 0.4938 & 0.0064 & 0.6516 & 0.0078 & 44.3641 & 0.5614 & 4223 & 19 & 3235 & 32 & 3873 & 14 & 84 & 325 & 11,453 & 5750 & 13,157 & 24,955 & 0.05652 & Inc & \\
\hline
\end{tabular}


Table 5. Cont.

\begin{tabular}{|c|c|c|c|c|c|c|c|c|c|c|c|c|c|c|c|c|c|c|c|c|c|}
\hline $\begin{array}{l}\text { Spot } \\
\text { No. }\end{array}$ & ${ }^{207} \mathrm{~Pb} /{ }^{206} \mathrm{~Pb}$ & $1 \sigma$ & ${ }^{206} \mathrm{~Pb} /{ }^{238} \mathrm{U}$ & $1 \sigma$ & ${ }^{207} \mathrm{~Pb} /{ }^{235} \mathrm{U}$ & $1 \sigma$ & ${ }^{207} \mathrm{~Pb} /{ }^{206} \mathrm{~Pb}$ & $1 \sigma$ & ${ }^{206} \mathrm{~Pb} /{ }^{238} \mathrm{U}$ & $1 \sigma$ & ${ }^{207} \mathrm{~Pb} /{ }^{235} \mathrm{U}$ & $1 \sigma$ & $\begin{array}{c}\text { Concordancy } \\
(\%)\end{array}$ & ${ }^{204} \mathrm{~Pb}$ & ${ }^{206} \mathrm{~Pb}$ & ${ }^{207} \mathrm{~Pb}$ & ${ }^{208} \mathrm{~Pb}$ & ${ }^{238} \mathrm{U}$ & ${ }^{204} \mathrm{~Pb} /{ }^{207} \mathrm{~Pb}$ & Inc/Exc & Notes \\
\hline 46 & 0.4894 & 0.0065 & 0.6799 & 0.0082 & 45.8711 & 0.5930 & 4210 & 19 & 3344 & 33 & 3907 & 15 & 86 & 279 & 10,540 & 5243 & 11,951 & 22,008 & 0.05321 & Exc & \\
\hline 47 & 0.8669 & 0.0088 & 0.2860 & 0.0031 & 34.1905 & 0.3687 & 5036 & 14 & 1622 & 16 & 3616 & 12 & 45 & 777 & 13,952 & 12,306 & 30,244 & 69,651 & 0.06314 & Exc & b \\
\hline 48 & 0.4972 & 0.0072 & 0.6791 & 0.0086 & 46.5568 & 0.6617 & 4233 & 21 & 3341 & 35 & 3921 & 16 & 85 & 313 & 9793 & 4954 & 11,138 & 20,592 & 0.06318 & Inc & \\
\hline 49 & 0.5108 & 0.0072 & 0.7374 & 0.0093 & 51.9359 & 0.7105 & 4273 & 21 & 3561 & 37 & 4030 & 16 & 88 & 197 & 7059 & 3669 & 8418 & 13,670 & 0.05369 & Exc & \\
\hline 50 & 0.5035 & 0.0068 & 0.6961 & 0.0086 & 48.3154 & 0.6385 & 4251 & 20 & 3406 & 34 & 3958 & 15 & 86 & 233 & 8236 & 4219 & 9461 & 16,898 & 0.05523 & Inc & \\
\hline 51 & 0.4086 & 0.0052 & 0.5346 & 0.0063 & 30.1152 & 0.3762 & 3941 & 19 & 2761 & 27 & 3491 & 14 & 79 & 235 & 12,837 & 5336 & 11,538 & 34,294 & 0.04404 & Inc & \\
\hline 52 & 0.4116 & 0.0064 & 0.5463 & 0.0070 & 31.0008 & 0.4509 & 3952 & 23 & 2810 & 30 & 3519 & 16 & 80 & 131 & 5235 & 2192 & 4653 & 13,684 & 0.05976 & Inc & \\
\hline 53 & 0.4689 & 0.0061 & 0.6022 & 0.0072 & 38.9287 & 0.4980 & 4146 & 19 & 3039 & 30 & 3744 & 14 & 81 & 221 & 9413 & 4490 & 10,079 & 22,322 & 0.04922 & Inc & \\
\hline 54 & 0.5037 & 0.0070 & 0.6836 & 0.0085 & 47.4700 & 0.6426 & 4252 & 20 & 3358 & 35 & 3941 & 15 & 85 & 185 & 6693 & 3430 & 7921 & 13,983 & 0.05394 & Inc & \\
\hline 55 & 0.4660 & 0.0062 & 0.5967 & 0.0072 & 38.3383 & 0.4977 & 4137 & 20 & 3017 & 31 & 3729 & 15 & 81 & 228 & 9452 & 4481 & 10,003 & 22,618 & 0.05088 & Inc & \\
\hline 56 & 0.4682 & 0.0068 & 0.6175 & 0.0077 & 39.8614 & 0.5506 & 4144 & 21 & 3100 & 32 & 3767 & 16 & 82 & 169 & 6028 & 2871 & 6551 & 13,941 & 0.05886 & Inc & \\
\hline 57 & 0.5054 & 0.0081 & 0.6809 & 0.0092 & 47.4539 & 0.7169 & 4257 & 23 & 3348 & 37 & 3940 & 17 & 85 & 128 & 5101 & 2623 & 6203 & 10,697 & 0.04880 & Inc & \\
\hline 58 & 0.4745 & 0.0062 & 0.6141 & 0.0074 & 40.1715 & 0.5132 & 4164 & 19 & 3086 & 31 & 3775 & 14 & 82 & 271 & 10,198 & 4922 & 11,044 & 23,715 & 0.05506 & Inc & \\
\hline 59 & 0.4109 & 0.0053 & 0.5469 & 0.0064 & 30.9847 & 0.3917 & 3950 & 19 & 2812 & 28 & 3519 & 14 & 80 & 207 & 11,033 & 4612 & 9957 & 28,810 & 0.04488 & Inc & \\
\hline 60 & 0.4873 & 0.0063 & 0.6545 & 0.0078 & 43.9712 & 0.5584 & 4203 & 19 & 3246 & 32 & 3865 & 14 & 84 & 276 & 9859 & 4887 & 11,148 & 21,511 & 0.05648 & Inc & \\
\hline 61 & 0.5060 & 0.0064 & 0.6879 & 0.0082 & 47.9915 & 0.6050 & 4259 & 19 & 3375 & 33 & 3952 & 14 & 85 & 312 & 11,239 & 5786 & 13,189 & 23,331 & 0.05392 & Inc & \\
\hline 62 & 0.4921 & 0.0061 & 0.6461 & 0.0076 & 43.8357 & 0.5412 & 4218 & 18 & 3213 & 31 & 3862 & 14 & 83 & 289 & 12,589 & 6302 & 14,254 & 27,823 & 0.04586 & Inc & \\
\hline 63 & 0.8656 & 0.0088 & 0.2867 & 0.0031 & 34.2216 & 0.3698 & 5034 & 14 & 1625 & 16 & 3616 & 12 & 45 & 759 & 13,760 & 12,118 & 29,798 & 68,525 & 0.06263 & Exc & $\mathrm{b}$ \\
\hline
\end{tabular}

Notes: a: Age ratios too high; b: High ${ }^{204} \mathrm{~Pb}$ cts. 


\section{Discussion}

\subsection{Advantages of the Mixed-Solution Method and Comparison of Data with GJ-1 Zircon}

Dating of the same hematite sample from the Olympic Dam high-grade ore (OD-10), using the GJ-1 Zircon calibration and the U-Pb solution method, produced ages that are statistically identical (1577 $\pm 5 \mathrm{Ma}$ and $1595 \pm 18 \mathrm{Ma}$ ). The larger error using the mixed-solution method is most likely attributable to the reduced size of the dataset (all 15 data points obtained are displayed in Figure 5, whereas 116 spots were included, and 97 rejected by Ciobanu et al. (2013)) [3]. Statistical overlaps between ${ }^{207} \mathrm{~Pb} /{ }^{206} \mathrm{~Pb}$ weighted average ages (using GJ-1 Zircon) and U/Pb upper intercept ages (using the $\mathrm{U}-\mathrm{Pb}$ mixed solution method) demonstrate that, although fractionation associated with a non-matrix matched standard does occur when using zircon as the primary standard, it does not impact the ${ }^{207} \mathrm{~Pb} /{ }^{206} \mathrm{~Pb}$ or intercept age. GJ-1 Zircon can thus be considered reliable for dating hematite by LA-ICP-MS. The data acquired in this study via the mixed solution method minimises these matrix effects and is considered more reliable since all analyzed points could be included.

The dates obtained for sample PH93 also overlap statistically (1597.2 \pm 6.1 Ma vs. $1604 \pm 11 \mathrm{Ma}$ when using GJ-1 Zircon and mixed solution standards, respectively). The larger error for the mixed-solution method can again be attributed to the smaller dataset. Of importance here is the fact that, unlike the U-Pb mixed-solution date, the ${ }^{207} \mathrm{~Pb} /{ }^{206} \mathrm{~Pb}$ GJ-1 Zircon age features data points that plot very far along the discordia (Figure 5). This clearly highlights the reduction in matrix effects by using the mixed-solution method. Both examples of high-U hematite show that the mixed-solution method used here can give higher quality data from the majority of analyzed points.

An important result of this study is that when using either the GJ-1 Zircon calibration or the solution method for the same samples (high-U, Olympic Cu-Au Province hematite), the majority of data plot either below or above the concordia. In both cases, however, data points that intercept the concordia are also obtained. Data points above concordia can be attributed to an increase in the ${ }^{206} \mathrm{~Pb} /{ }^{238} \mathrm{U}$ ratio due to downhole fractionation effects (Figure 7), a phenomenon also observed in zircon spot analysis by Paton et al. (2010) [19]. In this study, such an effect cannot be corrected for via modeling of the downhole fractionation whilst ablating the standard, as the $\mathrm{U}$ and $\mathrm{Pb}$ analyzed is not contained within the pure $\alpha-\mathrm{Fe}_{2} \mathrm{O}_{3}$ matrix ablated, but rather introduced as a solution, and thus the $\mathrm{U} / \mathrm{Pb}$ ratio remains unaffected by laser induced fractionation during ablation (Figure 7). The results here also place further constraints on the GJ-1 Zircon calibration method, where data points plotting below concordia can be mostly attributed to effects of matrix mismatch (different levels of $\mathrm{U}$ fractionation between zircon and hematite). In both cases, however, there is uncertainty about the relative loss or addition of $U$ (open system behavior), which cannot be solved by either method.

By including the low-U concentration Brazilian sample (6 ppm) in our study (CUR-002), we have shown that the solution method has advantages over the GJ-1 Zircon calibration method, and has wide application to analysis of samples containing low concentrations of $U$ (tens of ppm or less). Following this, it is reasonable to assume that samples of comparable $U$ concentration can be dated by this method, opening up considerable scope for very low $U$ age determinations elsewhere.

Although downhole fractionation was likely occurring during analysis of the CUR-002 sample, the large spread through the discordia (Figure 6) is predominantly due to the high amount of common $\mathrm{Pb}$ contained within these grains, as attested to by the high ${ }^{204} \mathrm{~Pb}$ measured during analysis. Of importance here, is that we have shown how samples of such low $\mathrm{U}$ concentration, can only be considered suitable for dating through the $\mathrm{U}-\mathrm{Pb}$ solution method, which can be tailored to accommodate the significantly lower U concentrations. This is because GJ-1 Zircon and other zircon standards have much higher U concentrations, relative to this sample, or others with similarly low-U concentrations, creating potential matrix effects when employed as the primary standard. 


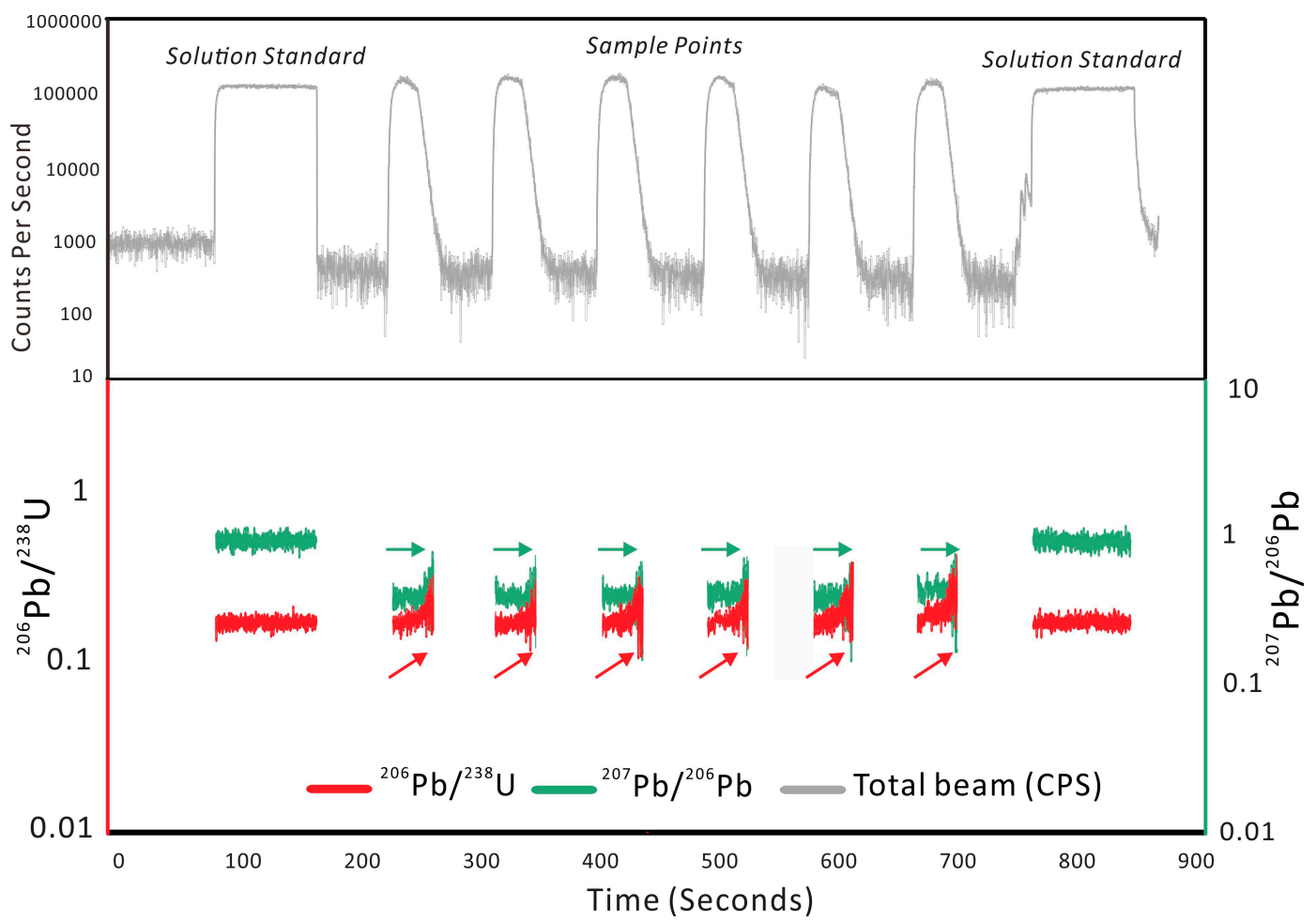

Figure 7. Time-resolved spectra data produced using IOLITE from data obtained from hematite in Olympic Cu-Au Province samples. Note no downhole fractionation within the solution standard, but significant fractionation of the ${ }^{206} \mathrm{~Pb} /{ }^{238} \mathrm{U}$ ratio (red), which increases with time during the duration of the spot analysis. The ${ }^{207} \mathrm{~Pb} /{ }^{206} \mathrm{~Pb}$ ratio (green) remains relatively constant throughout analysis, showing the robustness of the ${ }^{207} \mathrm{~Pb} /{ }^{206} \mathrm{~Pb}$ ages. This represents a minor disadvantage of the method but may be overcome in the future through linear rastering rather than spot analysis, if the homogeneity of the analyzed grain allows this.

\subsection{Geologic Meaning of the Hematite Ages}

The geological significance of the Olympic Dam hematite ages has been amply discussed in [3] and references therein, and supports formation of IOCG systems in South Australia coeval with LIP magmatism (Hiltaba Intrusive Suite and Gawler Range Volcanics) at 1.6 Ga.

In contrast to relatively abundant data for the Olympic Dam District, supportive geochronological data for other deposits in the Olympic $\mathrm{Cu}$-Au Province deposit remains sparse. The 1597.2 + 6.1 Ma hematite date obtained using GJ-1 Zircon as the primary standard does not overlap with a published $\mathrm{U} / \mathrm{Pb}$ zircon crystallization ages of $1586 \pm 3 \mathrm{Ma}$ for an associated granite from the central Mount Woods Inlier [20], but does statistically overlap with the of $1587 \pm 4$ Ma date obtained for a cumulate gabbronorite [20]. Although the mixed-solution $\mathrm{U}-\mathrm{Pb}$ hematite date $(1604 \pm 11 \mathrm{Ma})$ is statistically different to published ages, it is nonetheless very close. Minor differences in absolute age could be attributed to a non-direct relationship between the hematite associated with mineralization and LIP magmatism in the area. Most importantly, the present data for PH93 is further argument for the importance of the 1.6 Ga IOCG event throughout the Olympic Cu-Au Province.

The date obtained for the Brazilian sample $(1707 \pm 39 \mathrm{Ma})$ is within the range of geological events associated with the Carajás mineral province $(1.80 \mathrm{Ga} \mathrm{U} / \mathrm{Pb}$ zircon ages from A-type magmatism at the Carajás Granite [16], and 1.61 Ga U/Pb zircon ages from rift-related granitic magmatism [17]). The hematite dated here could represent a vein-related mineralizing event, but needs to be corroborated by data on samples with less common Pb or by using dating methods, such as ID-TIMS, for more accurate age determination. Regardless, the date obtained in this study is similar to the other ages considered for the protracted tectono-magmatic history of the Carajás mineral province [21]. 


\section{Implications and Outlook}

This study has successfully established a new matrix-matched dating method for hematite using a U-Pb mixed solution/pure hematite standard. Through the decreased matrix effects, homogeneity of the solution and tailoring of $U$ concentration differences between the sample and standard, a set of reliable dates have been obtained. The Fe-oxide hematite can now be dated using a U-Pb mixed solution/pure hematite standard method that provides high signal stability and has broad application to low-U samples. The samples will be further evaluated by ID-TIMS, to show whether the apparent discordance is due to the problems associated with the analytical methods used so far (e.g., Matrix-effects and downhole fractionation) or open system behavior. Although the zircon standardized data does show increased $\mathrm{U} / \mathrm{Pb}$ fractionation, most likely due to matrix effects, the upper intercept ages are still statistically the same as that of the solution method, making the GJ-1 Zircon standard reliable for obtaining upper intercept and ${ }^{207} \mathrm{~Pb}-{ }^{206} \mathrm{~Pb}$ weighted average ages. Using the mixed solution method, we have also validated the original age obtained for OD-10 [3]. We have demonstrated that although the use of GJ-1 Zircon as the primary standard does create more "apparent" fractionation with greater spreading of analytical points down the discordia, the upper intercept and weighted average ${ }^{207} \mathrm{~Pb}^{206} \mathrm{~Pb}$ dates obtained are still geologically meaningful. To eliminate downhole fractionation associated with the solution method, we will analyze samples using a line raster. This was not carried out in the analytical session described in this paper because of the lack of current samples exhibiting the same high U zonation patterns with large enough grains to carry out this type of analysis.

Further research includes finding suitable material for a solid hematite standard so that other more-precise microbeam methods, including SHRIMP, can be routinely used for dating. The mixed solution standard method presented here will also be applied to U-bearing magnetite following similar procedures. The petrographic and genetic link between Fe-oxides and hydrothermal mineralization is one that can be directly correlated when compared to other mineral geochronometers, such as monazite and zircon, making this study an important step towards establishing $\mathrm{U}-\mathrm{Pb}$ iron-oxide geochronology as a valuable tool that can be routinely used to constrain the genesis of mineral deposits.

Supplementary Materials: The following are available online at www.mdpi.com/2075-163X/6/3/85/s1, Supplementary Text File S1: Additional analytical procedures for calculating and certifying the $\mathrm{Pb} / \mathrm{U}$ ratios; Table S1: Pb isotope ratios of $\mathrm{Pb}$ standard solution; Table $\mathrm{S} 2: \mathrm{U}$ and $\mathrm{Pb}$ concentrations in standard solutions; Table S3: Correlation coefficient of the regression line.

Acknowledgments: This work is a contribution to the "FOX" project (Trace elements in iron oxides: deportment, distribution and application in ore genesis, geochronology, exploration and mineral processing), supported by BHP Billiton Olympic Dam and the S.A. Mining and Petroleum Services Centre of Excellence. Alexandre R. Cabral gratefully acknowledges VALE S.A. for financing his research position at the Technische Universität Clausthal and for logistically supporting his fieldwork in Carajás. We also gratefully acknowledge Yang Tao and Yan Xiong for their assistance with lead isotope analysis at the State Key Laboratory for Mineral Deposits Research, Department of Earth Sciences, Nanjing University, China.

Author Contributions: Zhiyong Zhu conceived and designed the experiments; Liam Courtney-Davies, Zhiyong Zhu and Cristiana L. Ciobanu performed the experiments under the guidance of Benjamin P. Wade. Liam Courtney-Davies, Zhiyong Zhu and Benjamin P. Wade processed the data; Kathy Ehrig and Alexandre R. Cabral contributed samples and information; Liam Courtney-Davies, Zhiyong Zhu, Cristiana L. Ciobanu and Nigel J. Cook wrote the paper, assisted by Benjamin P. Wade, Allen Kennedy and Kathy Ehrig. The work is part of the PhD project of Liam Courtney-Davies. We would also like to thank two anonymous reviewers for their detailed thoughts and important amendments, which helped us to clarify our interpretations, along with Matthew Horstwood with whom conversations further aided this study.

Conflicts of Interest: The authors declare no conflict of interest. The project sponsors approve publication of the manuscript.

\section{References}

1. Jackson, S.E.; Pearson, N.J.; Griffin, W.L.; Belousova, E.A. The application of laser ablation inductively coupled plasma-mass spectrometry to in-situ U-Pb zircon geochronology. Chem. Geol. 2004, 211, 47-69. [CrossRef] 
2. McFarlane, C.R.M.; Luo, Y. U-Pb Geochronology Using $193 \mathrm{~nm}$ Excimer LA-ICP-MS Optimized for In-Situ Accessory Mineral Dating in Thin Sections. Geosci. Can. Anal. Tech. Ser. 2012, 39, 158-172.

3. Ciobanu, C.L.; Wade, B.P.; Cook, N.J.; Schmidt Mumm, A.; Giles, D. Uranium-bearing hematite from the Olympic Dam Cu-U-Au deposit, South Australia; a geochemical tracer and reconnaissance $\mathrm{Pb}-\mathrm{Pb}$ geochronometer. Precambr. Res. 2013, 238, 129-147. [CrossRef]

4. Johnson, J.P.; Cross, K.C. U-Pb geochronological constraints on the genesis of the Olympic Dam Cu-U-Au-Ag deposit, South Australia. Econ. Geol. 1995, 90, 1046-1063. [CrossRef]

5. Günther, D.; Heinrich, C.A. Enhanced sensitivity in laser ablation-ICP mass spectrometry using helium-argon mixtures as aerosol carrier. J. Anal. At. Spectrom. 1999, 14, 1363-1368. [CrossRef]

6. Becker, J.S. State-of-the-art and progress in precise and accurate isotope ratio measurements by ICP-MS and LA-ICP-MS. Plenary Lecture. Anal. At. Spectrom. 2002, 17, 1172-1185. [CrossRef]

7. Günther, D. Laser Ablation-Inductively Coupled Plasma Mass Spectrometry Trends. Anal. Bioanal. Chem. 2002, 372, 31-32. [CrossRef]

8. Horn, I.; Rudnick, R.L.; McDonough, W.F. Precise elemental and isotope ratio determination by simultaneous solution nebulization and laser ablation-ICP-MS: application to U-Pb geochronology. Chem. Geol. 2000, 164, 281-301. [CrossRef]

9. Košler, J.; Fonneland, H.; Sylvester, P.; Tubrett, M.; Pedersen, R.-B. U-Pb dating of detrital zircons for sediment provenance studies-A comparison of laser ablation ICPMS and SIMS techniques. Chem. Geol. 2002, 182, 605-618. [CrossRef]

10. Simonetti, A.; Heaman, L.M.; Hartlaub, R.P.; Creaser, R.A.; MacHattie, T.G.; Böhm, C. U-Pb zircon dating by laser ablation MC-ICP-MS using a new multiple ion counting Faraday collector array. J. Anal. At. Spectrom. 2005, 20, 677-686. [CrossRef]

11. Leach, J.J.; Allen, L.A.; Aeschliman, D.B.; Houk, R.S. Calibration of Laser Ablation ICP-MS Using Standard Additions with Dried Solution Aerosols. Anal. Chem. 1999, 71, 440-445. [CrossRef]

12. Pickhardt, C.; Becker, J.S.; Dietze, H.J. A new strategy of solution calibration in laser ablation inductively coupled plasma mass spectrometry for multi element trace analysis of geological samples. J. Anal. Chem. 2000, 368, 173-181. [CrossRef]

13. Van Achterbergh, E.; Ryan, C.; Jackson, S.; Griffin, W. Appendix 3 Data reduction software for LA-ICP-MS. In Laser-Ablation-ICPMS in the Earth Sciences; Sylvester, P., Ed.; Mineralogical Association of Canada: Quebec City, QC, Canada, 2001; Volume 29, pp. 239-243.

14. Ludwig, K.R. A User's Manual for Isoplot 3.75: A Geochronological Toolkit for Microsoft Excel; Berkeley Geochronology Centre Special Publication No.5; Berkeley Geochronology Centre: Berkeley, CA, USA, 2012.

15. White, W.M.; Albarède, F.; Télouk, P. High-precision analysis of Pb isotope ratios by multi-collector ICP-MS. Chem. Geol. 2000, 167, 257-270. [CrossRef]

16. Machado, N.; Lindenmayer, Z.; Krogh, T.E.; Lindenmayer, D. U-Pb geochronology of Archean magmatism and basement reactivation in the Carajás area, Amazon Shield, Brazil. Precambr. Res. 1991, 49, 329-354. [CrossRef]

17. Pimentel, M.M.; Heaman, L.; Fuck, R.A.; Marini, O.J. U-Pb zircon geochronology of Precambrian tin-bearing continental-type acid magmatism in central Brazil. Precambr. Res. 1991, 52, 321-335. [CrossRef]

18. York, D. Least-squares fitting of a straight line with correlated errors. Earth Plan. Sci. Lett. 1969, 5, 320-324. [CrossRef]

19. Paton, C.; Woodhead, J.D.; Hellstrom, J.C.; Hergt, J.J.; Greig, A.; Maas, R. Improved laser ablation U-Pb zircon geochronology through robust downhole fractionation correction. Geochem. Geophys. Geosyst. 2010, 11, 1-36. [CrossRef]

20. Jagodzinski, E.A. Compilation of SHRIMP U-Pb geochronological data, Olympic Domain, Gawler Craton, South Australia, 2001-2003. Geosci. Aust. Rec. 2005, 20, 211.

21. Pinheiro, R.V.L.; Holdsworth, R.E. Reactivation of Archaean strike-slip fault systems, Amazon region, Brazil. J. Geol. Soc. 1997, 154, 99-103. [CrossRef]

(C) 2016 by the authors; licensee MDPI, Basel, Switzerland. This article is an open access article distributed under the terms and conditions of the Creative Commons Attribution (CC-BY) license (http://creativecommons.org/licenses/by/4.0/). 